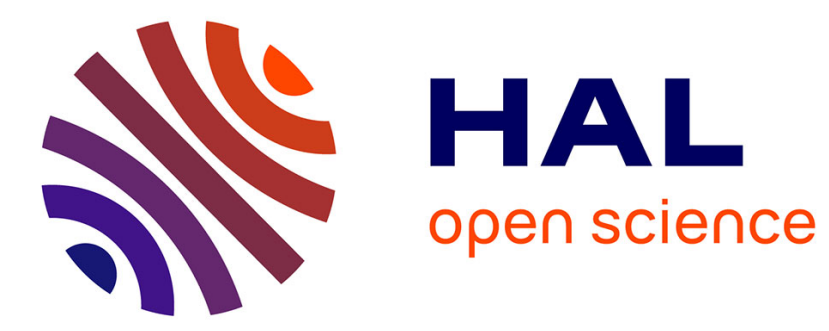

\title{
Perception de l'espace chez les notaires de Lucques (VIIIe-IXe siècles)
}

\author{
Anne Mailloux
}

\section{To cite this version:}

Anne Mailloux. Perception de l'espace chez les notaires de Lucques (VIIIe-IXe siècles). Mélanges de l'École française de Rome - Moyen Âge, 1997, 109 (1), pp.21-57. 10.3406/mefr.1997.3547 . halshs00505147

\section{HAL Id: halshs-00505147 https://shs.hal.science/halshs-00505147}

Submitted on 6 Feb 2017

HAL is a multi-disciplinary open access archive for the deposit and dissemination of scientific research documents, whether they are published or not. The documents may come from teaching and research institutions in France or abroad, or from public or private research centers.
L'archive ouverte pluridisciplinaire HAL, est destinée au dépôt et à la diffusion de documents scientifiques de niveau recherche, publiés ou non, émanant des établissements d'enseignement et de recherche français ou étrangers, des laboratoires publics ou privés. 


\section{Perception de l'espace chez les notaires de Lucques (VIIle-IXe siècle) \\ Anne Mailloux}

\section{Résumé}

Anne Mailloux, Perception de l'espace chez les notaires de Lucques (VIIle-IXe siècle), p. 21-57.

Les actes des archives de l'archevêché de Lucques fournissent des données précises sur la perception de l'espace chez les notaires lucquois des VIIle et IXe siècles. Dans un paysage où la centuriation antique est encore très tenace, ils décrivent les propriétés selon un système double, par genre de parcelle et par confronts. L'analyse de ces descriptions et du système métrologique relatif permet de reconstituer des pratiques d'arpentage révélatrices, si on les met en relation avec ce que l'on sait de la culture de ces notaires (Codex 490, comparaison des formulaires notariaux employés et des textes gromatiques antiques), de l'ancrage de la pratique notariale dans un bain culturel et technique hérité de l'Antiquité, et adapté aux contraintes juridiques et économiques lombardes et carolingiennes. Les

notaires font figure d'arpenteurs et de dépositaires de la mémoire foncière du territoire.

\section{Citer ce document / Cite this document :}

Mailloux Anne. Perception de l'espace chez les notaires de Lucques (VIIle-IXe siècle). In: Mélanges de l'Ecole française de Rome. Moyen-Age, tome 109, n¹. 1997. pp. 21-57;

doi : $10.3406 /$ mefr.1997.3547

http://www.persee.fr/doc/mefr_1123-9883_1997_num_109_1_3547

Document généré le 12/06/2016 


\section{PERCEPTION DE L'ESPACE CHEZ LES NOTAIRES DE LUCQUES (VIII ${ }^{\mathrm{e}}-\mathrm{IX}^{\mathrm{e}}$ SIÈCLE)}

L'analyse des dynamiques économiques et sociales du haut Moyen Âge, qui se développent dans un contexte essentiellement rural et agraire, s'appuie actuellement sur l'histoire des paysages, considérée comme le prolongement naturel de l'histoire des campagnes. On assiste depuis peu à un renouveau de ce type d'approches, ainsi que l'a souligné très récemment J.L. Abbé1. Il faut ici rappeler l'émergence de ces thèmes dès les travaux de Marc Bloch, dans le cadre d'une approche quasi organique de l'espace médiéval, qui permet de souligner la "naissance" des terroirs et du village comme expression spatialisée, "en dur", de la société rurale et médiévale. Ce regain d'intérêt pour l'objet rural se manifeste aussi dans la création de nouvelles revues, de groupes de travail interdisciplinaires, qui mettent au service de ces analyses des approches complètement renouvelées ${ }^{2}$. L'analyse s'est enfin nourrie des définitions du concept de paysage, sous l'influence des géographes, du moins dans le contexte français. Dans le domaine précis de l'histoire économique, mais aussi sociale, des campagnes, ces apports et croisements sont essentiels.

Ce champ d'études, délaissé pendant un certain temps, ou abordé de manière épisodique, a parallèlement été vivifié par un souffle nouveau, venu de l'archéologie, et notamment de l'archéologie extensive et de son croisement avec les sources écrites ${ }^{3}$. C'est elle qui apporte des éléments neufs,

' Cf. J.L. Abbé, Permanences et mutations des parcellaires médiévaux, dans Les formes du paysage. 2. Archéologie des parcellaires, Actes au colloque d'Orléans (mars 1996), G. Chouquer (éd.), Paris, 1996.

${ }^{2}$ Voir en premier lieu l'introduction de G. Brunel et J.-M. Moriceau, Un renouveau pour l'histoire rurale, dans Histoire et sociétés rurales (dorénavant H.S.R.), 1, $1^{\text {er }}$ semestre 1994, p. 7-10, et 2, 2c semestre 1994, p. 7-10, et l'article programmatique de M. Arnoux et G. Brunel, Réflexions sur les sources médiévales de l'histoire des campagnes, dans H.S.R., 1, $1^{\text {er }}$ semestre 1994, p. 11-35. La diversité des articles et études publiées depuis dans cette revue est la preuve éclatante de la vivacité de ce mouvement.

${ }^{3}$ Cf. Castrum II. Structures de l'habitat et occupation du sol dans les pays mé-

MEFRM - 109 - 1997 - 1, p. 21-57. 
concrets et précis sur les cultures, l'aspect global des paysages, voire la forme des parcellaires. Pour qui se livre à un inventaire bibliographique, c'est dans ce domaine que les publications récentes sont les plus nombreuses, les études de cas les plus fournies en volume. Le plus souvent, elles opèrent un croisement systématique entre la documentation écrite (actes privés, sources narratives...), les représentations figurées (cartes, cadastres...) et les résultats de la prospection archéologique (repérages de sites, analyses de la végétation, des types de culture...). On remarque d'ailleurs, pour ces enquêtes très serrées autour d'un territoire, un gonflement significatif de la documentation : l'analyse précise d'un espace, la mise en lumière de ses évolutions induisent une augmentation quasi exponentielle des renseignements nécessaires à sa bonne compréhension. La très belle étude d'A. Querrien sur un espace rural du Berry ${ }^{4}$ en est un exemple parfait : la justesse du propos s'allie à l'exhaustivité des renseignements tirés des sources employées, et à la diversité des techniques d'analyse. Quelques études très récentes des parcellaires, des structures de l'espace, de leurs représentations ou de leurs évolutions, sont les derniers jalons de ces enquêtes : il faut citer ici des ouvrages et articles publiés très récemment, ou en cours de publication, comme les travaux de M. Bourin, J.-M. Martin et L. Feller ${ }^{5}$.

Enfin, les recherches sur les cadastres et les centuriations romaines ont nettement influencé ce champ d'études. Les travaux qui se développent depuis une trentaine d'années dans ce domaine ont notamment permis de mieux appréhender les formes du paysage, passant de l'analyse descriptive à l'explication de la genèse des paysages agraires, dans le monde méditerranéen du moins, et dans celui des plaines, où se développèrent de manière

diterranéens : les méthodes et l'apport de l'archéologie extensive, G. Noyé (éd.), Rome (Collection de l'École française de Rome, 105), 1988.

${ }^{4} \mathrm{Cf}$. A. Querrien, Parcellaires antiques et médiévaux du Berry, dans Le Journal des savants, juillet-décembre 1994, p. 235-366, et compte rendu de M. Arnoux, dans H.S.R., 3, 1er semestre 1995, p. 338-339.

${ }^{5} \mathrm{Cf}$. M. Bourin, Délimitation des parcelles et perception de l'espace en Bas-Languedoc aux $X^{e}$ et $X I^{e}$ siècles, dans Campagnes médiévales: l'homme et son espace. Etudes offertes à Robert Fossier, E. Mornet (éd.), Paris, 1995, p. 73-85; J.-M. Martin, Perception et description du paysage rural dans les actes notariés sud-italiens (IX'-XII siècles), dans Castrum V, Actes du colloque de Murcie (1992) à paraître; L. Feller, Les Abruzzes médiévales. Territoire, économie et société en Italie centrale du IXe au XII siècle (thèse d'habilitation dactylographiée, Université de Paris I, 1996). Je remercie particulièrement J.-M. Martin et L. Feller de m'avoir confié leurs œuvres, et surtout de m'avoir prodigué avec générosité leurs conseils, idées et soutien pour cet article. Ma gratitude va évidemment à mon directeur de recherches, P. Toubert, pour m'avoir encouragée à ouvrir cette enquête. 
privilégiée ces opérations d'emprise de l'espace, au départ conçues comme des divisions à usage fiscal, mais qui eurent inévitablement des conséquences sur les formes matérielles de l'espace agraire, au point de le marquer de manière durable et pérenne ${ }^{6}$. Il suffit ici de rappeler la permanence des marques de centuriation, notamment dans les plaines italiennes, où des témoins encore très vifs de ces opérations deux fois millénaires sont toujours en place ${ }^{7}$. Les récentes études de parcellaires médiévaux soulignent invariablement leur dette vis-à-vis de ces recherches, ainsi que la permanence de ces structures dans les paysages du haut Moyen Âge ${ }^{8}$.

L'apport des recherches sur les parcellaires antiques, très influencées par l'étude des centuriations, permet de soulever des questions de fond; d'une part, il s'agit de placer le débat dans la perspective de la continuité discutée des paysages et donc de délimiter avec soin les phases d'évolution des parcellaires : comment expliquer la permanence plus ou moins forte des paysages antiques, où se placent, éventuellement, leur transformation et la formation de campagnes qui ont perduré?

Nous tenterons donc ici de fournir quelques éléments de réflexion, sur

${ }^{6}$ L'enquête menée en Italie centro-méridionale par G. Chouquer, F. Favory, M. Clavel-Lévêque montre à quel point les centuriations romaines sont encore visibles dans les espaces de plaine de la Campanie. Le réseau orthogonal imprimé par les Romains à la surface de la terre est parfois encore très nettement visible sur les cartes topographiques contemporaines. C'est ainsi le cas à Lucques. Cf. G. Chouquer, M. Clavel-Lévêque, F. Favory, J.-P. Vallat, Structures agraires en Italie centroméridionale. Cadastres et paysages ruraux, Rome (Collection de l'École française de Rome, 100), 1987.

${ }^{7}$ La bibliographie sur ce thème est extrêmement développée, et ne pourrait être épuisée dans l'espace d'une note de bas de page. Signalons toutefois quelques publications récentes : bibliographie commentée dans la contribution de J. Cortadella, L'histoire de la recherche sur les cadastres romains, dans De la terre au ciel, I: Paysages et cadastres antiques (XI ${ }^{\text {e }}$ stage international, Besançon, 29-31 mars 1993), M. Clavel-Lévêque, I. Jouffroy et A. Vignot éd., Besançon, 1994; G. Chouquer et F. Favory, Les paysages de l'Antiquité. Terres et cadastres de l'Occident romain, Paris, 1991. Du même G. Chouquer, un article de réflexion, qui effectue un retour sur l'historiographie de ces dernières années : Aux origines antiques et médiévales des parcellaires, dans H.S.R., 4, 2e semestre 1995, p. 11-46.

${ }^{8}$ Ainsi, M. Bourin écrit, dans l'introduction de son article : "Que reste-t-il au Languedoc de la science des arpenteurs romains lorsque recommence, après la longue interruption du très haut Moyen Âge, la conservation des documents écrits?", art. cit. supra note 5, p. 73 , et L. Feller, à propos de la diversité des formulaires notariaux dans la zone de la Conca Peligna, ne «voi $(\mathrm{t})$ pas d'autre explication à cette diversité qu'un effet lointain de la centuriation. La Conca Peligna est l'une des seules parties des Abruzzes dont nous soyons sûrs qu'elle a été soumise à cette institution. (...). Les repères antiques ont pu demeurer (...)», op. cit. supra note 5, p. 332. 
le passage d'un paysage antique, très fortement articulé par ces mêmes centuriations ${ }^{9}$, au paysage du haut Moyen Âge, sinon modifié profondément dans ses structures, du moins décrit selon un mode différent. Bien entendu, ces questions rejoignent, en aval, celles qui sont posées par le processus de l'incastellamento, entendu ici comme concentration de l'habitat, volontaire et dirigée, autour de centres seigneuriaux, parallèle à une réorganisation des terroirs ${ }^{10}$. Il eut sans conteste des conséquences sur la figure des campagnes italiennes. L'article d'A. Querrien souligne aussi le fait, en montrant que la période médiévale est l'occasion d'un réaménagement des campagnes et notamment d'un glissement progressif de la forme du parcellaire, d'un quadrillage hérité des centuriations, vers un parcellaire radioconcentrique, articulé de manière très forte autour des centres seigneuriaux ". Très nettement, ici, l'évolution matérielle des formes du parcellaire est à mettre en connexion avec les évolutions sociales et économiques médiévales, mais aussi avec celles des formes d'encadrement territorial. L'enjeu est par conséquent de définir les paysages, leurs dynamiques et leurs évolutions, comme révélateurs d'un mouvement, ou pour le moins d'une évolution des structures portantes de la société médiévale.

Pour le haut Moyen Âge lucquois, ces éléments revêtent une importance cruciale : nous nous plaçons ici en plein débat sur la continuité avec le monde antique, d'une part par proximité chronologique, d'autre part, parce que nous sommes dans un contexte géographique où la présence des Romains était prépondérante. Si la reconstitution des parcellaires antiques semble réalisable, par croisement de diverses méthodes d'investigation (de la télédétection à la fouille en milieu rural) ${ }^{12}$, le problème ne se pose pas de la même manière pour le Moyen Âge : l'absence de renseignements maté-

${ }^{9}$ Cf. F. Castagnoli, La centuriazione di Lucca, dans Studi Etruschi, XX, 19481949, p. 285-290; P. Sommella et C. F. Giuliani, La pianta di Lucca romana, dans Quaderni dell'Istituto di topografia antica dell'Università di Roma, VII, Rome, 1974, et A. Mailloux, Pour une étude des paysages à Lucques, dans De la terre au ciel, I : Paysages et cadastres antiques, op. cit. supra note 7, p. 207-222.

${ }^{10} \mathrm{Cf}$. P. Toubert, Les structures du Latium médiéval. Le Latium méridional et la Sabine du IXe siècle à la fin du XII' siècle, Rome (B.E.F.A.R., 221), 1973. Plusieurs bilans et réflexions méthodologiques ont depuis été menées sur le thème. Cf. en premier lieu C. Wickham, L'incastellamento ed $i$ suoi destini, undici anni dopo il Latium di P. Toubert, dans Castrum II : Structures de l'habitat et occupation du sol dans les pays méditerranéens : les méthodes et l'apport de l'archéologie extensive, Rome-Madrid (Collection de l'École française de Rome, 105; Casa de Velázquez, Série archéologie, IX), 1988, p. 411-420.

${ }^{11}$ Cf. A. Querrien, art. cit. note 4.

${ }^{12}$ Cf. les travaux de l'équipe de Besançon et, d'autre part, les éléments récents apportés lors du colloque d'Orléans, cf. supra note 1. 
riels très sûrs (il n'y a pas encore eu, hormis le travail d'A. Querrien, d'études fondées sur la détection de parcellaires médiévaux) est parfois compensée par la présence de sources écrites, décrivant par le menu les parcelles ou les biens fonciers, notamment grâce à la conservation précoce d'archives notariées. On dispose ainsi d'un autre type de renseignements : il est possible de déterminer le système de perception et de description de l'espace, hors du cadre omniprésent des opérations cadastrales romaines. D'un point de vue matériel, les données fournies sont moins directement transposables (sur une carte topographique ou sur une matrice cadastrale) que les éléments engendrés par les centuriations ${ }^{13}$. La méthode archéologique et l'analyse des sources écrites ont parfois du mal à se croiser, notamment à cause d'une différence majeure d'échelle d'appréhension des phénomènes : la centuriation se développe à l'échelle d'un territoire, la parcelle décrite dans une transaction a pour extension quelques centaines de mètres carrés.

\section{LE PAYSAGE LUCQUOIS : QUELQUES LIGNES DE FORCE}

A Lucques, dont la plaine constitue le terrain de notre enquête, la précocité et l'abondance des sources écrites d'une part, l'existence attestée d'une centuriation romaine encore visible de l'autre dessinent des conditions particulièrement favorables pour comprendre les systèmes de représentation de l'espace rural chez les "notaires" lucquois des VIII' et $\mathrm{IX}^{\mathrm{e}}$ siècles.

\section{Topographie}

Le territoire du diocèse de Lucques présente des caractéristiques assez classiques dans le monde italien ${ }^{14}$ : il s'agit d'une petite plaine bordée de collines, puis de monts, qui s'élèvent par endroits très brutalement. Ainsi,

${ }^{13}$ J'ai moi-même exprimé certaines nuances à ce sujet, cf. art. cit. supra note 9.

${ }_{14}$ Pour une meilleure approche des caractéristiques du paysage à Lucques, il faut se reporter à une bibliographie assez hétérogène et diffuse : voir, en premier lieu, C. Wickham, Economic and Social Institutions in Northern Tuscany in the $8^{\text {th }}$ Century, dans C. Wickham, M. Ronzani, Y. Milo, A. Spicciani, Istituzioni ecclesiastiche della Toscana medioevale, Galatina, 1980, p. 7-34; Id., The Mountains and the City. The Tuscan Appennines in the Early Middle Ages, Oxford, 1988. Sur la centuriation, F. Castagnoli, art. cit. supra note 9 et P. Sommella, C.F. Giuliani, op. cit. supra note 9. Il faut ajouter à ces ouvrages, qui proposent une bibliographie fournie, P. Squatriti, Water, Nature and Culture in Early Medieval Lucca, dans Early Medieval Europe, vol. 4, 1, 1995, p. 21-40, qui présente une belle approche des problèmes posés 
la limite sud du territoire sert de frontière naturelle avec la cité voisine de Pise, et les premiers contreforts des Apennins, avec la Garfagnana, qui comprend la haute vallée du Serchio, constituent un rempart très net vers le nord.

Le Serchio est d'ailleurs le second élément structurant la plaine de Lucques : il débouche d'un parcours très encaissé dans sa haute vallée sur une plaine à très basse altitude, largement ouverte, ce qui a conditionné par le passé l'obligation de réguler les variations de ce cours d'eau, d'autant plus que son écoulement inférieur, à l'issue de la plaine de Lucques, est aussi barré par un goulot d'étranglement naturel, la barre montagneuse des collines pisanes qui viennent s'éteindre justement en bordure sud et ouest de la plaine ${ }^{15}$. Les conditions hydrographiques ainsi définies engendrent un paysage de plaine placé sous la domination de l'eau : ruisseaux, rigoles de drainage, petits cours d'eau adjacents au fleuve, mais aussi marécages et lacs sont autant de repères incontournables dans la plaine.

\section{Mise en valeur}

Sur cette première trame, faite de contraintes naturelles, viennent s'imposer les réseaux mis en place par les habitants de la région : en termes d'habitat et de répartition de la population, mais aussi d'aménagement de l'espace agraire, l'épisode déterminant et "fondateur» est inévitablement l'opération de centuriation relative à la fondation de la colonie romaine de Luca vers 180 av. J.-C. La plaine est aujourd'hui encore marquée par cette trame qui a déterminé le tracé des chemins et voies de communication internes, mais aussi toute la "résille» hydrographique (les grandes voies de communication la traversent sans adaptation spécifique de la centuriation à ces tracés).

Quelques autres caractéristiques, relevant de la mise en valeur agraire du territoire, viennent compléter le tableau. En premier lieu, les cultures sont marquées par la domination de la vigne et de l'olivier, dans les zones légèrement surélevées, accompagnés par des prés, pacages, zones de fourrage, vergers, jardins. Cette diversité est enrichie par des champs de céréales. Deuxièmement, la dispersion de l'habitat, renforcée par la multitude de petites parcelles bien circonscrites, imbriquées les unes dans les autres, donne à la plaine de Lucques l'aspect d'une véritable mosaïque de

par la gestion de l'eau au Moyen Âge dans la plaine de Lucques, et A. Mailloux, art. cit. supra note 9.

${ }^{15}$ Cf. F. Castagnoli, art. cit. supra note 9, et D. Barsocchini, Sull'antico corso del Serchio, dans Atti della $R$. Accademia lucchese, 14, 1853, p. 391-487. 
cultures et de végétation... On relève ces éléments dans les contrats du haut Moyen Âge, où ils sont soigneusement notés (nous y reviendrons) ${ }^{16}$. Il est en revanche bien difficile de déterminer si ces caractéristiques existent déjà à l'époque romaine : si la centuriation et ses modules sont bien repérés, on n'a aucun renseignement sur les cultures pratiquées, pas plus que sur les structures de l'habitat. Les campagnes archéologiques font ici cruellement défaut ${ }^{17}$.

\section{Equilibres territoriaux}

Le dernier élément notable est la très forte polarisation qu'opère la ville, véritable foyer des activités économiques et sociales. Il existe de très forts liens entre la cité et la campagne environnante depuis l'Antiquité : une preuve pour cette époque semble fournie par la concordance de la déduction de la colonie de Lucques et de l'opération de centuriation. Le réseau urbain antique et la trame de la centuriation sont en effet tracés selon les mêmes axes. On constate, sur un autre plan, des relations cité/territoire semblables pour la période lombarde : bon nombre de propriétaires fonciers habitent en ville mais maîtrisent parfaitement leurs propriétés rurales sur l'ensemble de la plaine et du territoire de Lucques; les transactions foncières sont réalisées et enregistrées très régulièrement dans la cité18, alors qu'elles concernent plus souvent des biens situés dans l'ensemble du territoire.

\section{SOURCES}

Ce tableau brossé à la hâte est nécessaire à une meilleure compréhension des sources que nous allons examiner. En effet, le problème que nous nous posons est le suivant : quel est le système descriptif choisi par les notaires lucquois au très haut Moyen Âge lorsqu'ils enregistrent des transac-

${ }^{16} \mathrm{C}$. Wickham s'est ainsi livré à l'exercice de mesurer la proportion de chaque type de culture dans les actes lucquois, ainsi que leur répartition spatiale sur le territoire du diocèse. Cf. C. Wickham, Economic and Social Institutions, art. cit. supra note 14.

${ }^{17}$ On peut toutefois citer quelques études archéologiques, malheureusement très ponctuelles, et peu sensibles à ce type de préoccupations. Cf. G. Ciampoltrini et M. Zecchini, Capannori. Archeologia nel territorio, Lucques, 1987.

${ }^{18}$ Sur les lieux d'émission des actes, voir P. Supino Martini, Le sottoscrizioni testimoniali al documento italiano del secolo VIII : le carte di Lucca, dans Bollettino dell'Istituto storico italiano per il Medio Evo e Archivio Muratoriano, 98, 1992, p. 87-108. 
tions foncières et quelle est, par conséquent, leur perception de l'espace rural et agraire? Outre la compréhension du système descriptif choisi par les notaires, il nous faudra non seulement évaluer leur connaissance de quelques techniques d'arpentage et de mesure de la terre, et donc tenter de résoudre le difficile problème des mesures agraires, mais aussi savoir quelle est leur formation en la matière.

\section{Constitution du fonds}

L'Archivio arcivescovile di Lucca conserve une masse de documents incomparable par la richesse de renseignements qu'ils fournissent sur l'aspect des paysages au haut Moyen Âge. Ces archives sont particulièrement importantes pour qui s'intéresse aux modes de constitution d'un fonds notarié pour le haut Moyen Âge. Il faut ici souligner, en premier lieu, le cas exceptionnel que représente Lucques : la quantité de documents conservés est étonnamment élevée pour cette période et surtout, il n'y a pas de rupture chronologique dans la série des chartes conservées. De plus les actes, établis à la demande des propriétaires ou des locataires de terres, sont écrits par des notaires, selon des procédures claires et bien codifiées, et conservés le plus souvent dès leur établissement près de la cathédrale de Lucques $^{19}$. En cela, les Lucquois suivent les injonctions de la loi lombarde, qui exige très précisément un enregistrement des transactions. Dès Liut-

${ }^{19}$ Pour plus de précisions sur les pratiques notariales lucquoises, voir $P$. Supino Martini, art. cit. supra note 18. Pour l'étude des formulaires, voir aussi F. Martinelli, Le pergamene dell'Archivio arcivescovile di Lucca dall'anno 851 all'anno 864, dans Actum Luce, II, 1, Lucques, 1973, p. 105-114, sur les actes du IX' s. A titre d'exemple dans les sources, cf. l'acte de l'Archivio n ${ }^{\circ} 177 / 178$ (+G51, ++O60), du 16 mars 778 : «Et pro confirmatione duas cartulas uno tenore Filippum Subdiaconum nostrum scribere commonuemus. Actum in Domo Sancte Lucensis Ecclesie, de quas una esse decrevimus in Arcivo hujus S. Ecclesie, (...)". AAL 177 (+G51), Bert. IV I 86, Bars. V II 170, ChLA 1065; AAL 178 (++O60), Bars. V II 170, ChLA 1066 (a. 778). Pour les renvois aux sources, nous utiliserons dorénavant le système suivant : numéro de l'acte selon son classement actuel dans les Archives, suivi de sa cote, puis des renvois aux diverses éditions : Bars. V II : Barsocchini (D.), Memorie e documenti per servire all'istoria del ducato di Lucca, tome V, volume II, Lucques, 1837 (réimpression anastatique, Lucques, 1971), suivi du numéro dans l'édition; Bert. IV I : Memorie e documenti per servire all'istoria del ducato di Lucca, tomo IV, Lucques, 1818; Bert. IV II : ibid., Supplemento al tomo IV, Lucques, parfois suivi de A : Appendice (réimpression anastatique des deux volumes, Lucques, 1994), suivi du numéro dans l'édition. La plupart des actes édités par Bertini sont recensés dans l'édition Barsocchini, sous un numéro correspondant à leur place dans l'ordre chronologique (choisi par Barsocchini). Éditions récentes : CDL : Codice diplomatico Longobardo, éd. L. Schiaparelli, Rome, 1929-1933 et C.R. Brühl, Rome, 1974 (Fonti per la storia d'Italia, 62 - 
prand en effet, donc au tout début du VIII ${ }^{\text {e }}$ siècle, plusieurs chapitres de la loi lombarde concernent directement la procédure d'établissement des actes et rappellent la nécessité de produire des témoins ${ }^{20}$. De même, la possession d'un acte écrit devient une nécessité en cas de contestation de la propriété d'un bien. La procédure d'ostensio chartae devient la plus courante pour prouver un droit, ou rappeler des transactions précédentes. Dans les actes de jugement, l'importance de l'acte écrit, produit pour preuve, est constamment rappelée, y compris dans les notitiae iudicati produites et conservées à Lucques ${ }^{21}$.

La constitution du fonds et la conservation des actes ne sont donc pas accidentelles, ni ne résultent d'une entreprise postérieure de copie des actes (constitution de cartulaire, par exemple, comme c'est le cas à Farfa, pour justifier la possession de propriétés de l'abbaye). On dispose donc à Lucques d'un très grand nombre d'originaux, parfois même d'actes en double (un pour l'auteur, un pour les archives ${ }^{22}$ ), ou d'actes de même teneur juridique, mais contenant des formulaires différents (chartes de concessions mutuelles ${ }^{23}$ ).

La plupart des actes conservés dans le fonds de l'Archivio arcivescovile di Lucca, sont constitués par les titres de propriétés et les documents de gestion propres à l'évêché. Des transactions entre particuliers lui ont été adjointes par versement lors de l'intégration de ces biens dans le patrimoine ecclésiastique. A la fin du $\mathrm{X}^{\mathrm{e}}$ siècle, lorsque la fonction notariale est arrivée au terme de sa laïcisation ${ }^{24}$, et qu'elle est assumée par des membres

64); ChLA : Chartae Latinae antiquiores, éd. A. Bruckner et R. Marichal, vol. XXX à XL : Lucques, 1988-1991. Ces deux éditions ne couvrent pas le IX' siècle.

${ }^{20}$ Cf. Liut. 7 (a. 717), Liut. 22 (a. 721), Liut. 54 et Liut. 63 (a. 724), et Liut. 91 (a. 727), cf. C. Azzara et S. Gasparri, Le leggi dei Longobardi. Storia, memoria e diritto di un popolo germanico, Milano, 1992. Pour ce qui concerne l'histoire du notariat au haut Moyen Âge, cf. M. Amelotti, G. Costamagna, Alle origini del notariato italiano (Studi storici sul notariato italiano, II), Rome, 1975 et L. Schiaparelli, Note diplomatiche sulle carte longobarde, dans Archivio storico italiano, n. s. VII, XVIII, 1932, p. 334.

${ }^{21}$ Cf. à titre d'exemple AAL 303 (++K36), Bars. V II 289, ChLA 1184 (a. 800) : "Ecce cartulam per quam iste Altiprandus medietatem ex rebus ipsis offeruit (...)". Cet acte a aussi été édité dans C. Manaresi, I Placiti del "Regnum Italiae» (Fonti per la Storia d'Italia, $\left.\mathrm{n}^{\circ} 92\right), 1955-1960$, sous le $\mathrm{n}^{\circ} 11$.

${ }^{22}$ Cf. AAL 177/178 (+G51, ++O60), cf. supra note 18.

${ }^{23}$ Cf. AAL 175/176 (+O58/*L80), Bert. IV I 85 / Bars. V II 169, ChLA 1064/1063. Il s'agit d'une permutation de biens.

${ }^{24}$ Cf. H.M. Schwarzmaier, La Società lucchese nell'alto Medioevo e gli archivi ecclesiastici di Lucca, dans Archivi e Cultura, a. V. 1-2, janvier-décembre 1971. Cet auteur estime que le processus d'exclusion de clercs de la sphère des scribes et notaires 
de l'aristocratie urbaine, les archives ne sont plus seulement conservées aux archives épiscopales, mais aussi dans les offices notariaux privés ${ }^{25}$. Pour le $\mathrm{VIII}^{e}$ siècle et le début du siècle suivant, l'ambiguïté réside donc dans le fait que les notaires sont des ecclésiastiques rattachés à l'évêché, formés dans l'entourage épiscopal; l'évêque et l'administration épiscopale endossent en fait très souvent, à cette époque, une partie des fonctions administratives relatives au territoire de la cité.

Une dernière série de caractéristiques, quasi constantes dans le corpus d'archives, réside dans le fait que les actes sont passés à Lucques, ou dans son territoire ${ }^{26}$, établis par des notaires formés sur place, utilisant un stock de formules juridiques spécifiques et adaptées aux cas les plus fréquents qu'ils ont à traiter. Il est ainsi possible de reconstituer la carrière de certains notaires, mais aussi, étant donnée la grande homogénéité des formules, de saisir les éléments principaux de leur formation et les évolutions des pratiques juridiques et notariales en matière de transactions foncières.

\section{Typologie}

En effet, les chartes conservées concernent essentiellement des transactions foncières et décrivent par le détail les éléments nécessaires à l'opération : limites des parcelles, extension, type de culture (vignes, oliviers...), localisation, éléments de viabilité... Plusieurs types de description coexistent, répondant à des perceptions différentes de l'espace, dont nous nous attacherons à définir les grands traits et les principes.

Nous avons sélectionné, parmi les actes déposés à l'Archivio arcivescovile di Lucca, une série de documents suffisamment importante pour appuyer notre réflexion. Ils couvrent une période de plus d'un siècle, de 685 à $822^{27}$. 468 documents ont été examinés, dont quelques-uns enregistrent en

apparaît nettement vers 830 . Cf. aussi H. Keller, Der Gerichtsort in oberitalienischen und toskanischen Städten - Untersuchungen zur Stellung der Stadt im Herrschaftssystem des "Regnum Italicum" vom 9. bis 11. Jahrhundert, dans QFIAB, 49, 1969.

${ }^{25}$ Ces fonds notariaux ont par la suite été rapatriés à l'Archivio di Stato di Lucca.

${ }^{26}$ Sur la question des notaires «itinérants", voir les analyses très précises et intéressantes de P. Supino Martini, art. cit. note 18.

${ }^{27}$ La justification des limites chronologiques est exclusivement liee aux conditions de dépouillement aux archives : je n'ai ici pris en considération que les actes que j'ai pu vérifier, tant l'édition courante est lacunaire, notamment pour les renseignements qui nous intéressent : les descriptions des parcelles, avec limitations, sont parfois totalement passées sous silence, comme c'est le cas pour l'acte AAL 460 (++D32), Bert. IV II A 22, Bars. V II 445. 
fait plusieurs transactions, ou plusieurs étapes juridiques concernant un même bien, ou un même patrimoine, ou un même litige.

Parmi ces actes, 120 (soit à peu près un quart) comportent une description des biens avec indication des confins (par caput et latus) et/ou des surfaces. Ils se répartissent de la manière suivante :

\begin{tabular}{|c|c|c|c|c|c|c|c|}
\hline Date & Donation & Vente & $\begin{array}{c}\text { Échange } \\
\text { de biens }\end{array}$ & $\begin{array}{c}\text { Notice de partage, } \\
\text { de jugement }\end{array}$ & «Livello» & Prêt & Total \\
\hline $700-729$ & 1 & 1 & 0 & 0 & 0 & 0 & 2 \\
\hline $730-750$ & 2 & 6 & 1 & 0 & 0 & 0 & 9 \\
\hline $751-770$ & 8 & 6 & 3 & 4 & 2 & 0 & 23 \\
\hline $771-790$ & 9 & 7 & 6 & 1 & 3 & 0 & 26 \\
\hline $791-810$ & 11 & 8 & 8 & 2 & 5 & 0 & 34 \\
\hline $811-822$ & 6 & 2 & 5 & 6 & 4 & 3 & 26 \\
\hline Total & 37 & 30 & 23 & 13 & 14 & 3 & 120 \\
\hline
\end{tabular}

De manière globale, la typologie fait déjà ressortir quelques lignes de force, en matière de description des biens ${ }^{28}$ :

- Les transmissions de biens par donatio (ou offersio) forment un groupe important, suivies de près par les ventes et les échanges de biens (appelés viganeum ou cambiatio dans les formulaires des actes euxmêmes). Ces trois types de documents forment les trois quarts de la série sélectionnée.

- Les notices regroupent en fait plusieurs subdivisions typologiques, les notitiae brevis ou breve, qui inventorient, sur demande spécifique, des biens et possessions confirmés ou attribués auparavant, et les notitiae iudicati, qui sont des notes enregistrant le partage ou la répartition de biens, en prévision d'un héritage (ou après décès d'une personne. Il n'y a pas de véritable testament ${ }^{29}$ ). Nous les avons fondues en un seul ensemble, dans la

${ }^{28}$ Pour une comparaison avec la distribution générale des actes conservés à Lucques, voir F. Bougard, La justice dans le royaume d'Italie de la fin du VIII siecle au début du XIe siècle, Rome (B.E.F.A.R., 291), 1995, et notamment p. 97-98.

${ }^{29}$ Cf. E. Cortese, Il diritto nella storia medievale, $1:$ L'alto Medioevo, Rome, 1995. 
mesure où ces différents actes procèdent sensiblement de la même manière pour ce qui concerne la désignation des biens.

- Enfin, les contrats de location (livelli), ou les promissiones (attribution de biens liés à une église pour un recteur), et les quelques opérations appelées mutuo, prêts sur gage immobilier, semblent marginaux dans notre inventaire de 120 actes, ce qui ne reflète pas du tout la réalité de la distribution globale sur l'ensemble des 468 actes : si les contrats de prêt sont toujours très marginaux, les contrats de location et de concession de biens sont très nombreux, surtout à partir de la fin du VIII ${ }^{\text {e }}$ siècle. On peut en déduire a priori que le formulaire employé dans les concessions (notamment dans les livelli) ne ménage que très rarement une place à une description circonstanciée des parcelles. L'attention des contractants est plutôt sollicitée, dans ce cas, par d'autres éléments : obligations diverses et redevances sont ainsi clairement détaillées. La valeur foncière importe alors beaucoup moins.

\section{Cohérence}

Il convient de souligner en outre un élément technique assez révélateur : la très grande majorité des actes sélectionnés a été établie à Lucques, ou dans des lieux environnants, par des notaires lucquois, ou formés à Lucques. En effet, la plupart d'entre eux apparaissent plusieurs fois et les formulaires employés dans ce contexte sont d'une très grande régularité. En revanche, quelques très rares documents se singularisent par une différence du lieu d'émission, toujours liée à l'apparition d'un notaire ou scribe inconnu dans le contexte lucquois et à une très grande variation du formulaire, notamment en matière de description des biens ${ }^{30}$. Leur très faible nombre est le reflet de la cohésion et de la cohérence de l'atelier notarial lucquois, qui est le relais quasi exclusif lorsqu'il s'agit d'enregistrer des transactions. Malgré tout, ces quelques actes nous serviront à définir, en contrepoint et par comparaison, la manière qu'avaient les Lucquois de décrire leurs parcelles, et donc leurs connaissances des techniques métrologiques et agrimensorielles.

${ }^{30}$ Il s'agit des actes AAL no 46 (+74), Bert. IV I 45, CDL 111, ChLA 938 (a. 754); AAL n ${ }^{\circ} 146\left(^{*}\right.$ A14), Bars. 141, CDL 275 (a. 772); AAL n 335 (*G11), Bars. 319 (a. 805/ 806). 


\section{SYSTÈMES DE DESCRIPTION DES PARCELLAIRES}

S'attacher à un exposé sur les systèmes de description des biens fonds nécessite d'avoir une idée claire des éléments qui sont retenus comme nécessaires à l'identification des terres ainsi décrites. J.-M. Martin ${ }^{31}$ détermine deux critères : la description interne du bien et sa description externe. La démarche qu'il suit est extrêmement commode et a le mérite d'établir en fait une hiérarchie des critères qui semble exister dès l'époque, au point que leur succession est presque immuable : désignation de la parcelle, environnement (subdivisé en deux genres, topographie et confronts). Elle permet de mettre en évidence la grande variété et la grande souplesse de ce système descriptif, et par là sa profonde adaptation aux réalités matérielles de l'espace, ainsi que la précision des connaissances de détail des notaires et contractants.

\section{Description générique et désignation des parcelles}

Commençons donc par la désignation générique des biens. Nous devons d'emblée préciser un élément, de taille : dans la série de documents sélectionnés pour notre étude, nous avons exclu les documents présentant les biens composés d'éléments variés selon une description par genre. Ils représentent pourtant une masse importante, que nous n'aurions pas eu le temps d'analyser intégralement. Malgré tout, il convient de s'arrêter un instant sur ces transactions; il s'agit généralement d'opérations de divers types : donations, concessions, ventes et parfois (très rarement) échanges, portant sur des biens fonds et mobiliers, qui composent des ensembles cohérents. On pourrait multiplier à l'infini les exemples. Il faut retenir ici deux éléments fondamentaux et, en premier lieu, la variété de désignation des ensembles ainsi décrits, selon différents registres : res désigne le bien en général, sorticellula ou sorticella ${ }^{32}$, de même que portio ou conquisitio, désignent des modes d'acquisition (par héritage, ou par achat), casa ou sorte $^{33}$, curte $^{34}$, qui décrit plutôt un type de bien (exploitation cohérente, habitat...). La terminologie employée peut aussi indiquer un partage de l'ensemble, mais dont on se rend compte, à la lecture de la description de détail, qu'il compose un groupe cohérent et articulé de biens : "medietas de

${ }^{31}$ Cf. J.-M. Martin, art. cit. supra note 5.

${ }^{32}$ AAL 5 (+H84), Bert 35, ChLA 897, CDL 27 (a. 720).

${ }^{33}$ AAL 5 (+H84), Bert 35, ChLA 897, CDL 27 (a. 720), pour casa, voir aussi AAL 22 (+H94), Bert 40, ChLA 914, CDL 67 (a. 738) (morgengab); AAL 6/7 (+N4, ++O67), Bert 36, ChLA 898-899, CDL 28 (a. 720).

${ }^{34}$ AAL 6/7 (+N4, ++O67), Bert 36, ChLA 898-899, CDL 28 (a. 720). 
omnem ris mea vel conquisito meo" "35, "homnem res mea (...) quartam portionem " ${ }^{36}$, "sexta portione ex omnibus rebus meis» ${ }^{37}$. Le terme désignant l'ensemble des biens peut être encore plus général : res $^{38}$, ou au contraire précis, ecclesia ${ }^{39}$, ou enfin refléter des conditions d'occupation ou de concession (bref, une situation juridique particulière), terra vacua ${ }^{40}$, casas massaricias ${ }^{41}$.

Deuxièmement, la relative homogénéité des descriptions de biens est frappante, mais le formulaire n'est jamais figé ni redondant : on remarque ainsi des variations dans les termes employés qui prouvent, si besoin était, que la transaction s'établit sur des données précises et reflète une situation patrimoniale et domaniale, voire agricole, extrêmement bien maîtrisée :

"casa, vinea, servus vel ancilla, cultum, desertum, movile, inmovile, sequemoventibus ${ }^{42}$;

"(...) fundamentum cum curte, puteo, orto, aditu, accessu et egitu (...) sala cum duas casas tributarias (...) vinea, oliveto, silva, peculiare, prato" ${ }^{43}$.

Les éléments agricoles et végétaux sont ainsi assez précis :

"homnem res mea (...) quartam portionem, cum sis mancipiis (...) vinis pratis pascuis, silvis, salectis, pumiferis fructiferis, diversis territuris, movile vel inmovile vel seseque moventibus ${ }^{44}$;

"casa (...) cum omnia adiacentia sua, simol cum territoriis vineis olivetis, silvis, virgariis, cultis vel incultis, omnia et in omnibus, mobilia vel inmobilia seo semoventibus" ${ }^{45}$; "(...) vel omnia quidquid in meo dominio esset videtur, tam orto, fenile, casas masaricias (...) cum territurii, cultis et incultis (...) " ${ }^{46}$; "casa abitationis nostre cun fundamento curte (...) orto et alia ividem edificia, cum terris, vineis pratis pascuis silvis virgareis castanaetis cultum adque incultum, mobile vel inmobile seu semoventibus, omnia et in omnibus rebus nostris ${ }^{47}$.

${ }^{35}$ AAL 14 (+I59), ChLA 906, Bars 15, CDL 42 (a. 726-729).

${ }^{36}$ AAL 18 (+L20), ChLA 910, Bars 19, CDL 58 (a. 736, ou 741).

${ }^{37}$ AAL 67 (+N87), ChLA 960, Bert 53, CDL 145 (a. 760).

${ }^{38}$ AAL 25 (++068), ChLA 917, Bert 41, CDL 73 (02 740).

${ }^{39}$ Dans le cas d'une concession à un recteur, qui comprend non seulement la charge pastorale de l'église, mais aussi la gestion et la possession matérielle des biens, AAL $35(+\mathrm{N} 2)$, ChLA 927, Bars 37, CDL 89 (a. 747).

${ }^{40}$ AAL 25 (++068), ChLA 917, Bert 41, CDL 73 (a. 740).

${ }^{41}$ AAL 43 (+B71), ChLA 935, Bars 44, CDL 105 (a. 752).

42 AAL 5 (+H84), Bert 35, ChLA 897, CDL 27 (a. 720).

${ }^{43}$ AAL 8 (+K64), ChLA 900, Bars 10, CDL 30 (a. 722).

${ }^{44}$ AAL 18 (+L20), ChLA 910, Bars 19, CDL 58 (a. 736, ou 741).

${ }^{45}$ AAL 22 (+H94), ChLA 914, Bert 40, CDL 67 (a. 738).

${ }^{46}$ AAL 25 (++068), ChLA 917, Bert 41, CDL 73 (a. 740).

${ }^{47}$ AAL 82 (++M1), ChLA 975, Bars. 81, CDL 169 (a. 763). 
Dans ce dernier cas, l'énumération des biens est très standardisée et ne varie que sur un terme : "castanaetis", révélateur d'un milieu végétal particulier (en forêt relativement élevée, ce qui nous permet de mieux localiser le bien), et d'un type de production très spécialisé.

Dans le cas de transactions portant sur des parcelles dûment décrites, limitées et désignées, la terminologie employée ne varie guère, si ce n'est qu'elle traduit des ensembles plus restreints et circonscrits : terra, vinea, sont les éléments du parcellaire les plus couramment désignés, suivis par un ensemble précis désignant des types agricoles de parcelles : silva, pratum, olivetum, "cafagio", virgarium, "orto", prata, salecto ou saliceto, campus : cette diversité du lexique renvoie à la diversité du paysage agricole, où les cultures nécessitant l'usage ou la présence de l'eau sont bien représentées. On peut noter aussi la présence de parcelles en complant : «vinea cum arboribus", ce qui n'implique pas forcément une coltura promiscua. Sont également très employés les termes désignant de petites parcelles "petia", "petio», voire "pezo», attachés le plus souvent aux terres et vignes, mais aussi aux jardins. L'expression "petia de terra» doit toutefois être assez générale, et désigner (comme en français d'ailleurs), une pièce de terre, tout comme "petia de vinea». Les expressions "petio de terrula» et "petiolo de orto" désignent à coup sûr, quant à elles, de très petites parcelles. Cela nous permet de préciser qu'elles devaient malgré cela avoir une valeur marchande intéressante puisque leur existence est nettement rappelée. Autre terme rappelant des subdivisions de biens faisant l'objet d'une mise en valeur précise et importante pour l'époque : "portionem de silva». La diversité de la terminologie en ce qui concerne le couvert boisé (silva, cafagio) montre le soin apporté à la gestion de ces espaces au cours du haut Moyen Âge et la bonne connaissance qu'en avaient les notaires ${ }^{48}$.

L'éclatement de l'espace agricole en un microparcellaire très bigarré est aussi évoqué par des désignations d'éléments combinés : "terra et foscione", "terra et vinea", qui sont désignés comme des binômes groupés. Cet entrelacs de parcelles ressort encore plus lorsqu'on se penche sur les descriptions des confins des parcelles et la désignation des parcelles adjacentes : bien souvent, les parcelles jouxtant une parcelle décrite font l'objet d'une autre culture ${ }^{49}$. La mosaïque parcellaire est enfin complétée par l'imbrication et la dispersion de l'habitat dans la trame parcellaire : "clausu-

${ }^{48}$ Voir, entre autres, L. Lagazzi, Segni sulla terra. Determinazione dei confini e percezione dello spazio nell'alto medioevo, Bologna (Biblioteca di storia agraria medievale, 8), 1991.

${ }^{49}$ Voir l'exemple traité dans A. Mailloux, art. cit. supra note 9, p. 221 : deux cas, un pré entouré de terres (dont on ne désigne pas précisément l'occupation agricole), 
ra», ensemble enclos comprenant souvent maisons et petites parcelles ${ }^{50}$, mais aussi "casa et vinea", "casa et terra" (nous pouvons ici renvoyer au premier point que nous avons évoqué : descriptions génériques de patrimoines ou exploitations complètes, où l'habitat semble totalement intégré au parcellaire $)^{51}$. Notons au passage qu'hormis les cas de ventes de biens immobiliers en ville, il n'y a que très peu de maisons désignées comme limite à une parcelle : on peut probablement voir là un indice de la dispersion de l'habitat, au moins dans la plaine de Lucques. Enfin, une terminologie assez précise désigne l'habitat : clausura $^{52}$, casalino, sala, solario ${ }^{53}$, et prouve sa variété, de l'habitat intégré à l'espace agraire (clausura) à la caractéristique architecturale (solario). Le tableau ainsi brossé à l'aide des descriptions est composé d'un habitat largement dispersé, intégré dans un espace agraire sans homogénéité.

\section{Localisation et toponymie, topographie}

La localisation des parcelles fournit, somme toute, très peu de renseignements sur la topographie : les notaires indiquent en fait le plus souvent le toponyme en usage pour certaines zones et le nom du lieu (Lunata, par exemple). Peut-être faut-il voir ici le reflet de la topographie uniforme de la zone couverte par les actes : la plupart des transactions comprenant des descriptions précises de parcelles concernent la plaine. Très peu touchent les collines, encore moins la Garfagnana. Pas de précision dans la description du relief, donc, contrairement à ce qu'on peut no-

et une "vinea et salecto" entourée de vignes, terres et même d'un ruisseau (aqua qui percurrit da via pubblica). AAL n 376 (+B85), Bars. 360 (a. 808).

${ }^{50} \mathrm{Cf}$. la note de S. Gasparri, qui rappelle la teneur de la loi lombarde sur certains éléments de l'aménagement de l'espace agraire, particulièrement protégés par la loi, notamment les "enclos» (Rothari 29, 32 et 33). Cf. C. Azzara et S. Gasparri, op. cit. supra note 20, Introduction, p. XII. Voir aussi L. Lagazzi, op. cit. supra note 48. Dans cet ouvrage, consacré aux systèmes de marquage de la terre et des parcelles dans la plaine du Pô lombarde, L. Lagazzi relève, parmi les confins importants fréquemment cités dans les documents privés, les termes de clausura (enceintes), $c(a)$ esae (palissades), s(a)epes ou s(a)epta (haie), auxquels on peut ajouter incisae (probablement proche de caesa).

${ }^{51}$ Voir AAL $138(++\mathrm{N} 35)$, ChLA 1030, Bars 133, CDL 265 (a. 772); AAL 230 (+O21), ChLA 1117, Bars. 221 (a. 788); AAL $282(++$ G88), ChLA 1165, Bars. 269 (a. 798).

${ }^{52}$ Dans un certain nombre de cas, le terme de clausura ne désigne pas seulement un champ enclos, mais un ensemble plus complexe, enclos, comprenant une maison et de petites parcelles.

${ }^{53}$ Solario : maison à étage 
ter dans certains actes du Sud italien ${ }^{54}$. En revanche, on constate une certaine précision du vocabulaire lié à l'eau et au régime hydrographique de la plaine : fossa ${ }^{55}$ (ruisseau de drainage), servant fréquemment de limite, et parfois renforcé d'une haie : fossa et cesa ${ }^{56}$, foscionel fuscione ${ }^{57}$ (?), classo ${ }^{58}$ (chiasso?), aquis cum terminivas suis ${ }^{59}$, rio $^{60}$, ad Rivo ${ }^{61}$, parfois précisé par un toponyme, toujours en cours : Rio Fraga ${ }^{62}$, Rio Zana ${ }^{63}$, Rio Frachula ${ }^{64}, a$ rio Tiola ${ }^{65}$, fleuve ${ }^{66}$, qui sert parfois de point de repère précis pour localiser une parcelle : "trans Auserclo ${ }^{67}$. Quelques toponymes se rapportent directement à cet environnement : Ripariu ${ }^{68}$, Insula Surbanise ${ }^{69}$. On a même des éléments précis sur les formes d'aménagement des parcelles, et notamment du drainage : "padule suo usque in fossa" ${ }^{70}$.

Les chemins et routes servent aussi très souvent de limites : le terme le plus fréquemment employé est celui de via pubblica ${ }^{71}$. La fréquence de ces

${ }^{54}$ Cf. J.-M. Martin, art. cit. supra note 5, pour les actes de Cava dei Tirrenni : monte, collis, serra, ...

${ }^{55}$ AAL 52 (+H86), ChLA 944, Bert 49, CDL 125 (a. 757); AAL 114 (+Q82), ChLA 1006, Bars 112, CDL 227 (a. 769); AAL 230 (+O21), ChLA 1117, Bars. 221 (a. 788); AAL 241 ( ${ }^{\star}$ L76), ChLA 1128, Bert. 108 (a. 790).

${ }^{56}$ AAL 282 (++G88), ChLA 1165, Bars. 269 (a. 798), cf. L. Lagazzi, op. cit. supra note 48 , et $P$. Squatriti, art. cit. supra note 14.

${ }^{57}$ AAL 53 (+L6), ChLA 945, Bars 54, CDL 126 (a. 757).

${ }^{58}$ AAL 114 (+Q82), ChLA 1006, Bars 112, CDL 227 (a. 769).

${ }^{59}$ AAL 219 (+M39), ChLA 1105, Bars. 208 (a. 786).

${ }^{60}$ AAL $90(++\mathrm{H} 3)$, ChLA 982, Bert 59, CDL 179 (a. 764).

${ }^{61}$ AAL 128 r. ( ${ }^{\star}$ F90), ChLA 1020 a, Bert 72, CDL 250 (a. 771).

${ }^{62}$ AAL 148 (+O22), ChLA 1039, Bars 143, CDL 279 (a. 773).

${ }^{63}$ AAL 349 (+N64), Bars. 333 (a. 806).

${ }^{64}$ AAL $373(++\mathrm{G} 14)$, Bars. 357 (a. 808).

${ }^{65}$ AAL 447 (+Q86), Bars. 431 (a. 819).

${ }^{66}$ AAL 114 (+Q82), ChLA 1006, Bars 112, CDL 227 (a. 769); AAL 241 (*L76), ChLA 1128, Bert. 108 (a. 790).

${ }^{67}$ AAL $108\left({ }^{\star}\right.$ G91), ChLA 1000, Bert 63, CDL 214 (a. 768), le vocable d'Auserclo désigne l'ancien cours du Serchio. Voir, sur le cours du Serchio, F. Castagnoli, op. cit. supra note 9, et Barsocchini, Sull'antico corso del Serchio, art. cit. supra note 15.

${ }^{68}$ AAL $377(+\mathrm{N} 82)$, Bert. II 10, (a. 808) complété par mes soins.

${ }^{69}$ AAL 447 (+Q86), Bars. 431 (a. 819).

${ }^{70}$ AAL 342 (+H97), Bars. 327 (a. 806).

${ }^{71}$ AAL 88 (+N88), ChLA 980, Bars. 86, CDL 177 (a. 764); AAL 104 (+Q53), ChLA 996, Bars 102, CDL 205 (a. 767); AAL 106 ( ${ }^{*}$ E30), ChLA 998, Bars 104, CDL 210 (a. 767); AAL 114 (+Q82), ChLA 1006, Bars 112, CDL 227 (a. 769); AAL $163\left({ }^{\star} \mathrm{O} 11\right)$, ChLA 1053, Bars 156 (a. 775); AAL 241 ( ${ }^{\star}$ L76), ChLA 1128, Bert. 108 (a. 790); AAL $280(+\mathrm{O} 18)$, ChLA 1164, Bars. 267 (a. 798); AAL $282(++$ G88), ChLA 1165, Bars. 269 (a. 798); AAL 444 (+O83), Bars. 428 (a. 819), parmi d'autres. À propos de l'adjectif "public», il faut noter qu'il est fréquent, non seulement à Lucques, mais aussi dans 
limites permet de penser que la plaine de Lucques est extrêmement bien desservie, et que le réseau des chemins permet un très bon accès au parcellaire. D'ailleurs, on ne remarque pas, ou très peu, dans les actes, de cas d'appropriation privée du réseau viaire ${ }^{72}$, ou de cession de droits de passage, à travers des parcelles ou des propriétés, pour l'accès à un champ. Les mentions de droits d'entrée et de sortie sont limitées à des patrimoines plus étendus $^{73}$. Enfin, les haies et rideaux d'arbres servant de limites semblent très fréquents, ce qui est logique étant donné la nature de l'environnement que nous pouvons percevoir. Le terme de "semita» est souvent employé ${ }^{74}$, mais le vocabulaire ne se limite pas à cette seule forme, cesa apparaissant aussi parfois ${ }^{75}$.

Bref, la topographie est réduite ici à une seule forme d'expression : il suffit de localiser globalement une parcelle sur un territoire, mais surtout d'indiquer précisément les confins et confronts de la parcelle : le tracé des cours d'eau et des chemins sert ici à merveille de support à la description. Il semble donc qu'à Lucques, la limitation s'appuie très facilement sur des repères linéaires. Il convient toutefois, bien entendu, de relativiser cette affirmation : la limite peut aussi être à la fois ligne et espace, composée de haies et fosses, route. Malgré tout, l'aspect très géométrique de l'espace est un élément patent de la plaine de Lucques. Il ne faut pas oublier à ce propos que nous sommes dans un espace extrêmement anthropisé et maîtrisé : nous en voulons pour preuve la richesse de la toponymie, très pérenne d'ailleurs, mais aussi la persistance de la centuriation. On ne peut, arguant de la mention fréquente de toponymes et éléments relatifs au réseau hydrographique, affirmer que la plaine ne fait pas l'objet d'un aménagement spécifique et adapté aux conditions hydrographiques. On ne peut donc dresser le tableau épouvantable d'une région abandonnée, désertée et reconquise par les éléments naturels. L'eau est un élément omniprésent, mais très bien géré par les Lucquois du haut Moyen Âge ${ }^{76}$.

d'autres lieux : J.-M. Martin, art. cit. supra note 5. Les voies sont perçues soit comme des espaces, séparés des champs par des haies, soit comme un tracé linéaire. Il semble que ce soit aussi le cas à Lucques. Voir aussi L. Lagazzi, op. cit. supra note 48.

${ }^{72}$ Une mention de via non qualifiée de publique : Via Ropprandi, AAL 368 (+O74), Bars. 352 (a. 808).

${ }^{73}$ Cf. supra, acte cité, AAL 8, n. 43. Il s'agit, en plus, d'une mention unique, et précoce, dans la série de documents lucquois.

${ }^{74}$ AAL $90(++\mathrm{H} 3)$, ChLA 982, Bert 59, CDL 179 (a. 764).

${ }^{75}$ AAL 282, cf. supra note 51.

${ }^{76}$ Voir à ce propos P. Squatriti, art. cit. supra note 14 et A. Mailloux, art. cit. supra note 9 à propos notamment de la légende de saint Frediano. 


\section{Limites et confronts}

Espace anthropisé, espace maîtrisé : l'élément de description le plus frappant est l'énoncé des confronts, de la nature des limites matérielles et des parcelles adjacentes, le plus souvent définies par leur situation patrimoniale et agricole ${ }^{77}$.

\section{Caput/Latus}

Deux systèmes de délimitation des confronts sont en concurrence, dont l'un l'emporte très nettement sur l'autre : la description par "capita et latera" est très fréquente, alors que la délimitation par parcours est extrêmement rare, et liée à des opérations d'arpentage très particulières (division de biens, réattribution nécessitant le bornage du terrain...). La description par "capita et latera» laisse à penser que les parcelles décrites sont quadrangulaires, trapézoïdales mais surtout, et plutôt, rectangulaires ou carrées, en tout cas régulières : en effet, il semble que caput désigne le petit côté, et latus le côté le plus long ${ }^{78}$. Il n'y a que très peu de cas où les éléments confinant la parcelle dépassent le nombre de quatre, et dans ces caslà, on peut penser qu'un côté est bordé par deux parcelles elles-même adjacentes ${ }^{79}$.

L'emploi du binôme caput/latus, régulier et toujours dans le même ordre, semble indiquer, ici aussi, que ces deux termes servent à désigner des parcelles "orientées", en tout cas ayant une forme rectangulaire : on suit ici toujours un ordre logique, par paire de côtés opposés, d'abord les capita, puis les latera ${ }^{80}$. Très peu de cas dérogent à la règle : l'emploi du seul terme de caput et la description précise d'un seul, ou de deux côtés de la parcelle $^{81}$, sont très rares; l'emploi du seul terme de latus $^{82}$ nous semble

${ }^{77}$ Il en est à Lucques de même que dans l'Italie du Sud : les pièces sont le plus souvent définies par le nom de leur propriétaire, et la situation patrimoniale antérieure est parfois rappelée. L'acte sert donc aussi de mémoire des transactions antérieures. Cf. J.-M. Martin, art. cit., supra note 5.

${ }^{78} \mathrm{Cf}$. L. Lagazzi, art. cit. supra note 48 qui estime que la description des pièces de terre par capita et latera renvoie à un maillage régulier du parcellaire.

${ }^{79}$ On peut se reporter par exemple à la parcelle décrite dans AAL 376 (+B85), Bars. 360 (a. 808), schématisée dans A. Mailloux, art. cit. supra note 9, p. 221.

${ }^{80}$ Cf. J.-M. Martin, art. cit. supra note 5, "le système de description des confronts".

${ }^{81}$ AAL $15\left(++\right.$ O64), ChLA 907, Bert 37, CDL 48 (a. 730); AAL $108\left({ }^{\star}\right.$ G91), ChLA 1000, Bert 63, CDL 214 (a. 768); AAL $450(+51$ ), Bars. 434 (a. 820).

${ }^{82}$ AAL 280 (+O18), ChLA 1164, Bars. 267 (a. 798); AAL 289 (+O28), ChLA 1170, Bert. 120 (a. 799); AAL $162\left({ }^{\star}\right.$ A24), ChLA 1052, Bars 155 (a. 775); AAL $209(++$ F26), ChLA 1095, Bars. 200 (a. 785). 
moins significatif, dans la mesure où le terme désigne de manière générale le côté, sans préjuger d'une taille, ou d'une orientation particulière. Il ne prend de sens précis qu'en étant accolé à caput. Ce système d'organisation des confronts semble utilisé de manière exclusive à Lucques : on n'observe pas d'orientation par point cardinal (dans les descriptions de parcelles) ${ }^{83}$. C'est toujours par un tracé adjacent, ou par référence à une parcelle contiguë que la limite est désignée.

Enfin, l'origine même des termes de caput et latus renforce cette hypothèse. Rappelons à ce propos que l'ensemble des chercheurs qui se sont penchés sur ce problème établissent un lien très net entre la description par caput-latus et la référence au système technique d'arpentage romain. Les termes de caput et latus sont d'ailleurs directement tirés des écrits des arpenteurs romains : on peut par exemple citer le traité De iugeribus metiundis $^{84}$, où ces deux termes sont utilisés avec des acceptions assez proches du sens qu'ils ont dans les actes du haut Moyen Âge : latus pour côté, sans contestation possible, caput désignant plutôt "la tête», c'est-àdire l'extrémité étroite. Au haut Moyen Âge, caput désigne le côté étroit (la largeur), par opposition à latus, longueur, si on décrit une parcelle rectangulaire, par exemple ${ }^{85}$.

L'extrême régularité de ces descriptions, et la situation quasi exclusive des parcelles dans la plaine (centuriée, faut-il le rappeler?), laissent à penser que ces parcelles sont parfaitement intégrées dans le réseau orthonormé antique. La description par capita et latera semble donc réservée au milieu de la plaine ${ }^{86}$.

${ }^{83} \mathrm{Ce}$ qui semble au contraire être le cas, plus tardivement il est vrai, dans la zone salernitaine, au $\mathrm{X}^{\mathrm{e}}$ siècle, au détriment du système par capita et latera, alors abandonné (cf. J.-M. Martin, art. cit. supra note 5), et aussi dans le Languedoc à partir de la même période (cf. M. Bourin, art. cit. supra note 5, p. 74 et suivantes).

${ }^{84}$ Cf. De Iugeribus metiundis, (éd. J.-M. Martin et J.-P. Grélois), dans J. Lefort (dir.), Géométries du fisc byzantin, dans Réalités byzantines, 4, Paris, 1991.

${ }^{85} \mathrm{Cf}$. le sens de caput : "extrémité d'une parcelle de terre", relevé dans l'article caput, dans J.F. Niermeyer, Mediae Latinitatis Lexicon Minus, Leyde, 1976. Le sens de caput chez les gromatiques diffère, et désigne plutôt un angle. Mais on peut penser qu'il y a synonymie avec frons, qui prend nettement ce sens. C'est dans le corpus des gromatiques que ces termes ont les sens les plus proches des acceptions altomédiévales, et dans des inscriptions qui sont en fait des éléments de cadastres (C.I.L. III, 9315, 7, cippus prope Salonas), ce qui tendrait à renforcer l'idée que ce vocabulaire est très nettement lié à l'usage romain et aux centuriations.

${ }^{86}$ Rappelons ici la remarque de bon sens de J.-M. Martin : "on décrit de façons différentes les parcelles situées en terrain plat et en terrain accidenté ", art. cit. supra note 5 . 
Parcours, Arpentage, Bornage

Le second système de description procède d'un esprit totalement différent, qu'on pourrait qualifier de percursif : en effet, la description se fait alors en suivant le parcours des limites du terrain, ou de l'ensemble. C'est plutôt dans le cas de brefs que ce type de description se rencontre, en vue d'une division du bien. Le plus bel exemple fourni par les archives de Lucques est l'acte AAL 460, établi le 30 mai 822, dans la cité de Lucques $^{87}$, par un notaire local, Petrus clericus notarius. Dans cet acte sont concentrés nombre d'éléments complètement absents des descriptions par capita et latera : parcours orienté, avec des termes désignant des points cardinaux (aquilone, meridie, mais aussi da exurgente); aspect composite du patrimoine attribué dans l'acte : l'auteur procède en versant dans le patrimoine un grand nombre de parcelles, décrites les unes après les autres. Dans cette description, des points de repère servent à situer les parcelles : il s'agit essentiellement d'arbres (ad fico albo, ad ulmo trisile).

Malgré tout, nous sommes bien loin ici du système de description longuement analysé par L. Lagazzi, qui pense qu'il existe deux systèmes de perception de l'espace, l'un romain, l'autre lombard, radicalement différents, le premier fondé sur une division orthonormée de l'espace, le second

${ }^{87}$ AAL 460 (++D32), Bert. IV II A 22, Rég. Bars. V II 44 (a. 822). Malheureusement une bonne partie, la plus intéressante pour notre propos, n'est pas éditée dans l'ouvrage de Bertini. Or, le passage qu'il a omis de transcrire contient les éléments les plus intéressants pour nous. J'en transcris ici la teneur "ad Arme ipsa medietate da exurgente similiter et medietate de ille undecim petiole de terra nostra ad loco plutiano lato da exurgente et medietate de prato nostro qui dicitur ad Tertie lato da meridie seo et medietate de due petiole de terre nostre ad ispice ipsam medietas da exurgente et medietate de ille sex petiole de terre nostre qui dicitur ad ulmo trisile da exurgente itemque et medietate de terra nostra ad cafagio barucci lato da exurgente et medietate de terra nostra ad Rusignano lato da exurgente et medietate de terra nostra ad Sancto Georgio lato da exurgente et medietate de terra nostra da area noua lato da exurgente item et ponimus in ista sorte medietate de terra nostra qui dicitur clausura cicula lato da exurgente et medietate de ille due petie de terra nostra da Campo Gleuuli. Ipsa medietas da exurgente et medietate de ille due petiole de terra nostra da area Landuli lato da aquilone et medietate de campo nostro qui dicitur uinea Barucci lato da exurgente et medietate de campo nostro da area lato da exurgente et medietate de res petiole de terra nostra da isula lato da exurgente". Un deuxième acte contient des éléments similaires, avec mention des points cardinaux et pose de signes de confins, AAL $76(+\mathrm{L} 46)$, Bert. IV I 55, ChLA 969, CDL 161 (a. 762). Il s'agit, une fois de plus, d'un partage de biens. Cet acte contient en outre des mentions très précises de mesures de surface et de mesures linéaires, ce qui en fait un cas précieux pour nous. Il faut souligner encore le caractère exceptionnel de ce document, dans la mesure où il émane directement de l'évêque de Lucques, Peredeo, qui procède ainsi à un partage de son patrimoine, assez conséquent, il va sans dire. 
de type "périmétral" ${ }^{88}$. En effet, même dans ce document, on a une description des parcelles avec mention de capita et latera.

Quelques actes mentionnent en revanche des signes de confins différents des tracés que nous avons relevés précédemment : il ne s'agit plus de lignes, ou d'espaces contigus, mais d'éléments ponctuels, comme les arbres, cités ci-dessus, ou des signes non précisés, dont on peut penser qu'il s'agit de signes sur des arbres, des pierres, mais sans précision possible. L'expression employée est "finis signa posita". Dans ce cas-là, il semble bien que lors de l'établissement de l'acte, il y ait eu une opération de parcours, ou de reconnaissance, ou de marquage sur le terrain. L'expertise, en quelque sorte, est nécessaire pour renforcer la légitimité juridique de la division des biens.

Cette opération de délimitation, qui semble connue des notaires lucquois, et maîtrisée, voire enseignée et transmise par eux (les actes où ces expressions apparaissent sont distants de plusieurs dizaines d'années, 762, $822 \ldots$ ) ne me semble pas incompatible avec l'utilisation du système romain de confins. Ainsi, les opérations de bornage décrites dans le corpus des Agrimensores romains semblent prévoir la pose de signes divers, afin d'éviter notamment les conflits de bornage, liés au "déplacement» des limites ${ }^{89}$.

\section{Systèmes de description}

La typologie des descriptions semble conditionnée en premier lieu par des facteurs politico-culturels, comme le soutient L. Lagazzi, et comme le démontre J.-M. Martin, qui met clairement en évidence la variation des formulaires en fonction de la formation du notariat, nettement influencé par les techniques romaines dans certaines zones, et par les pratiques (si ce n'est un système de référence spatial complètement différent) lombardes

${ }^{88} \mathrm{Si}$ la démonstration semble extrêmement séduisante et fonctionne pour la curtis de Migliarina, on ne peut s'empêcher de penser que, même dans le système terminal en vigueur dans ce cas-là, les filiations avec le système romain sont assez importantes. Notamment, les signes de confins gravés dans le bois apparaissent dans la typologie des signes de confins établie par les arpenteurs romains. Cf. L. Lagazzi, art. cit. supra note 48.

${ }^{89}$ Quelques passages de l'article de J.-M. Martin concernent eux aussi le bornage. Il me semble toutefois que l'utilisation d'arbres comme bornes n'est pas l'appanage exclusif de zones boisées, ou peu mises en valeur. Dans la plaine de Lucques, où les arbres, sous toutes formes de types de plantation semblent nombreux, leur utilisation traduit une bonne anthropisation de l'espace. Une petite étude sur les conflits de bornage est enfin parue récemment : P. Jaillette, Les conflits de bornage dans le Code Théodosien. Textes et traduction, dans H.S.R., 2, 2e semestre 1994, p. 161-179. 
dans d'autres. Mais on ne peut se limiter à ce seul facteur de typologie. Nous aurions tendance à penser qu'elle est aussi déterminée par la fonction des actes ${ }^{90}$.

Dans la zone de Lucques, où le corpus d'archives, et donc le formulaire employé, sont cohérents ${ }^{91}$, où on ne peut par conséquent arguer de différences politico-culturelles majeures, on trouve en concurrence les deux systèmes (lombard et romain, pour simplifier). Force nous est donc de trouver une autre explication aux différents systèmes de description de l'espace agraire. Ceux que nous avons tenté de relever semblent répondre à plusieurs contraintes. D'une part, une très grande précision est nécessaire lors de transactions où la valeur marchande doit être évaluée au plus serré : c'est le cas par exemple des échanges de biens, où la parité de valeur des parcelles est incontournable. Dans le cas de ventes, la description matérielle et objective du bien est un élément préalable à la fixation du montant de la somme déboursée. D'autre part, dans les locations, il importe plutôt de donner une idée de la localisation du bien, de sa mise en valeur agricole et du revenu qu'on espère en tirer. Dans les partages de biens, l'aspect strictement patrimonial est souligné : délimitation et localisation, rappel de la situation juridique antérieure, et parfois, comme dans le cas d'autres transactions (vente, par exemple, où le titre de propriété doit être transmis intégralement), transmission de munimina. Ici, la valeur marchande du bien importe peu, il n'est donc pas forcément besoin de donner une indication de surface, par exemple.

\section{Métrologie}

Après avoir examiné les perceptions et les systèmes de description de l'espace en vigueur chez les notaires lucquois, il convient de dégager la finalité de ces descriptions. L'examen de la typologie des actes met nettement en évidence que ces documents ont pour but de conserver et de formaliser diverses transactions foncières, et que les notaires modulent leur formulaire en fonction de la nature de la transaction, de l'extension et de la composition du bien en question, et effectuent éventuellement des expertises d'arpentage différentes : délimitation par parcours, ou vérification des mesures, bornage. C'est donc dans un but éminemment pratique, celui de fixer une valeur économique à la terre, que les notaires se livrent à cette

${ }^{90}$ Cf. J.-M. Martin, art. cit. supra note 5 : «un même critère préside toujours à ce choix : celui de la précision juridique".

${ }^{91}$ Voir supra, la présentation des sources disponibles. 
description précise de l'espace. Plusieurs problèmes se posent dans ce contexte : il faut comprendre quelle est la technique de mesure des parcelles.

Le problème des mesures est extrêmement délicat à aborder, pour toute une série de raisons : d'une part, la tentation la plus fréquente est d'établir une équivalence entre le système métrologique employé et le système métrique actuel, afin d'obtenir une idée de la surface, et donc de l'importance des transactions en jeu ${ }^{92}$. Dans la plupart des cas, les traités de métrologie des $\mathrm{XIX}^{\mathrm{e}}$ et $\mathrm{XX}^{\mathrm{e}}$ siècles procèdent, dans cette visée, selon le mode suivant : relevé des mesures locales, avec analyse de leur appellation et de leur évolution chronologique ${ }^{93}$, continuité ou non avec le système romain... Pour fixer une valeur standard à certains modules de référence (comme le pied romain par exemple), on effectue une moyenne des mesures relevées dans différentes régions. C'est ainsi qu'on donne au pied romain la mesure standard de $0,295 \mathrm{~m}$ à peu près ${ }^{94}$, au pied de Liutprand une mesure qui tourne autour de 0,43 à $0,44 \mathrm{~m}$, soit l'équivalent du cubitus romain, qui représente 1,5 fois le pied romain. Ce type de démarche est relativement illusoire, dans la mesure où, s'il nous aide à nous représenter plus

${ }^{92}$ La bibliographie relative aux problèmes de métrologie peut se classer en deux grands groupes : certains ouvrages tendent à l'exhaustivité, et veulent dresser un tableau universel des mesures. Ce type d'entreprise a fréquemment eu lieu au XIX ${ }^{e}$ siècle, dans une perspective plus ou moins positiviste : voir en particulier A. Martini, Manuale di metrologia, ossia misure, pesi e monete in uso attualmente e anticamente presso tutti i popoli, Turin, 1883. Signalons, à titre de curiosité, M. Bertini, Delle lineri e itinerarie misure antiche e moderne. Tavola generale presentata da $M$. Bertini, dans Atti della $R$. Accademia lucchese, 6, Lucques, 1830, p. 265-340. Le second groupe est composé d'études de métrologie régionale, qui tentent d'éclaircir, à l'usage du néophyte, les subtilités et complexités de systèmes métrologiques locaux. Quelques-uns ont retenu notre attention, dans la mesure où ils permettaient de comprendre les systèmes métrologiques du haut Moyen Âge et leur articulation avec le système romain : A. Mazzi, Il sextarius Pergami. Saggio di ricerche metrologiche, Bergame, 1877. Id., Il piede Liprando e le misure di Garlenda, Bergame, 1885. Pour la région lucquoise : G. Cordero di San Quintino, Delle misure lucchesi e del miglior modi di ordinarle, dans Atti della Reale Accademia lucchese, 1, Lucques, 1819-1821, p. 1-28. Plus récemment, l'étude de V. Villani, Per una storia della metrologia agraria medievale. L'area umbro-marchigiana e la Marca d'Ancona, Serra de' Conti (Ancône), 1982, fournit une mise au point nécessaire sur la mise en place des systèmes métrologiques de l'époque lombarde.

${ }^{93} \mathrm{~V}$. Villani souligne ainsi la tendance à l'accroissement des modules métrologiques au cours du Moyen Âge, notamment pour le pied de Liutprand.

$940,2978 \mathrm{~m}$ chez Martini, qui est la référence la plus communément citée, $0,29423 \mathrm{~m}$ chez Mazzi. 
facilement les surfaces, il ne rend pas compte d'un élément fondamental : la variation, à l'intérieur d'un même système métrologique, et au cours d'une même période, des modules de base.

Au-delà de ce strict problème d'interprétation, il semble beaucoup plus important de comprendre la logique interne de chaque système métrologique, son fonctionnement et sa finalité. L'énoncé de mesures sert-il réellement à fixer une valeur à la terre?

\section{Pratiques d'arpentage}

Un premier point à résoudre est celui de savoir s'il existait de réelles opérations d'arpentage, assumées par un personnel spécialisé, ou occasionnel, formé peu ou prou à ce type d'activités. A priori, il n'y a pas de corps d'arpenteurs, spécifiquement désigné, formé et répondant à des règles professionnelles et juridiques définies, comme c'était le cas dans l'Antiquité. Mais, s'il n'existe pas d'arpenteurs, il semble toutefois que les notaires - à Lucques pour le moins - soient qualifiés - si ce n'est formés spécifiquement - à ce type d'opérations. À preuve, deux éléments :

- L'envoi de missi, épiscopaux pour la plupart, lors d'échanges de biens avec melioratio pour l'église. Le missus, qui est aussi notaire dans d'autres actes, est envoyé sur le lieu de la transaction pour vérifier la taille du bien que reçoit l'église, selon la prescription législative lombarde ${ }^{95}$. Il semble qu'à cette occasion, le missus se livre à une opération d'évaluation des superficies, assez précise pour que les mesures des parcelles soient indiquées et rappelées dans l'acte notarié. Bien entendu, ces mesures ne sont indiquées qu'en cas de transactions portant sur des parcelles de faible étendue, très nettement individualisées et limitées. Lorsqu'il y a des échanges de domaines cohérents, de patrimoines plus étendus et composites, ou très articulés (comprenant des biens mobiliers et immobiliers, des biens fonds...), ces indications de mesures n'ont plus lieu d'être. Le type d'évaluation des biens est alors différent.

Pour ce qui concerne les échanges de parcelles (et non plus de propriétés complètes), l'indication et la certification de la mesure jouent un rôle

${ }^{95}$ Sur cette particularité de la loi lombarde, voir C. Egger, Eine neuaufgefundene Privaturkunde des 10. Jahrhunderts aus Lucca, dans Mitteilungen des Instituts für österreichische Geschichtsforschung, 100, H. 1-4, 1992, p. 150-160. Il renvoie pour cette disposition à Aistulf 16. Cf. Cl. Azzara et S. Gasparri, op. cit. supra note 20, p. 259 : en fait, la loi n'exige pas de melioratio du bien attribué à l'église, mais uniquement la présence de missi. La plupart du temps, les échanges avec accroissement du bien pour l'église sont donc des donations cachées. 
fondamental dans la transaction : le soin apporté à la mesure semble indiquer qu'on se livre alors à une véritable opération d'arpentage et de mesure des champs. Cette hypothèse peut être vérifiée par la mention de la présence du missus sur le lieu de la transaction. On peut à titre d'exemple se reporter à la transaction, déjà citée, enregistrée dans l'acte $n^{\circ} 237^{96}$. Il s'agit d'un échange de bien avec melioratio. Le texte de l'acte comprend l'expression «melioratum cambium dedit ad partem prefate Ecclesie S. Fridiani quam recepissem». Dans les souscriptions apparaît celle de «Sinderadu clericus missus predicti Johanni episcopi sicut supra legitur interfui, manu mea subscripsi». La souscription de Sinderadu est autographe.

- Deuxièmement, les partages et descriptions de biens, en vue d'un héritage, laissent entrevoir un autre type d'évaluation et de mesure de la terre. Les parcelles sont ici décrites avec force détails, mention d'un parcours, de limite en limite, d'arbre en arbre, de repère en repère, avec parfois la mention d'un marquage spécifique des arbres (la teclatura par exemple) ${ }^{97}$. Dans ce cas aussi, il y a opération réelle d'arpentage, avec parfois mention de la surface des parcelles. Pour le moins, un parcours est effectivement réalisé pour pouvoir ensuite rendre compte de l'extension du bien dans l'acte notarié, qui dans ce cas a pour fonction d'enregistrer non seulement la transaction, mais aussi les formes matérielles de l'espace en jeu.

\section{Outils}

Dans ce contexte précis d'opérations d'évaluation foncière, la mention d'outils spécifiques à l'arpentage - la pertica (perche), par exemple, qui est l'objet le plus fréquemment cité - est très intéressante. On peut se poser bien des questions sur ce vocable : on ne sait s'il s'agit uniquement d'une mesure (héritée des Romains, et dont nous verrons si la valeur correspond ou non à la pertica romaine, et si oui, dans quelle mesure et dans quel rapport), ou bien aussi de l'outil servant à la mesure. L'expression "ad iusta pertica mensurata", ou "per mensura da iusta pertica $"^{98}$, accolée à l'indica-

${ }^{96}$ Cf. AAL 237 ( ${ }^{\star}$ E96), Bars. 228 (a. 789). L'agencement du parcellaire est schématisé dans A. Mailloux, art. cit. supra note 14, p. 220. L'écriture de Sinderadu apparaît aussi dans AAL 211 (+I56), ChLA 1097, Bert. 94 (a. 785) et elle semble une écriture de "qualité nettement supérieure", selon l'estimation de M. Palma, éditeur du volume 38 des ChLA. Sur les souscriptions autographes, cf. P. Supino Martini, art. cit. supra note 18.

${ }^{97}$ Teclatura : marquage du tronc d'un arbre sur le tronçon dont on a auparavant ôté l'écorce.

${ }^{98}$ AAL 41 (+G4), ChLA 933, CDL 102 (a. 752), AAL $400(++I 10)$, Bars. 384 (a. 813), "per mensura da iusta pertica», AAL 376 (+B85), Bars. 360 (a. 808). 
tion d'une superficie ou d'une mesure linéaire, semble induire une mesure physique et réelle de la parcelle, à l'aide de la perche de référence dans la zone. D'autre part, il n'y a aucune raison de ne pas supposer que, si ce terme désigne dans le monde romain antique l'outil et la mesure, il n'en est pas de même pour la période suivante.

\section{Mesures}

\section{Unités}

Nous avons constaté, dans notre dépouillement systématique, que certaines parcelles sont dotées d'indications de surface et de mesures linéaires. Le relevé systématique des mesures indiquées dans les transactions peut être ordonné selon le moyen suivant :

- mesures linéaires : pertica ${ }^{99}$;

- mesures de superficie : tabula ${ }^{100}$, iscala ${ }^{101}$;

- mesures de superficie du haut Moyen Âge, correspondant à des mesures de capacité ou à des mesures de surface établies de manière standardisée depuis l'époque romaine : modius, modilocus, sistaria ${ }^{102}$.

Cette apparente hétérogénéité des systèmes de mesures doit malgré tout être tempérée : les mesures en muids sont exprimées "ad iusta pertica mensurata ", ce qui laisse à penser que :

- dans l'esprit des notaires établissant l'acte, il existe un référent exact en surface pour le muid, qui n'est dès lors plus variable;

- qu'on sait passer de la perche au muid.

99 "Una pertica de latitudine", AAL 76 (+L46), ChLA 969, Bert. 55, CDL 161 (a. 762); AAL 386 ( $\left.{ }^{\star} E 14\right)$, Bars. 370 (a. 810), qui est un des seuls actes à comporter les mesures des côtés et qui, de manière révélatrice, n'a pas été établi à Lucques : «et est per mensura de uno latere perticas sex, de alio latere similiter, de capita sunt vero duos perticas per capu (...) de uno latere perticas septe et media, de alio sex et media, de uno capo pertica una, de alio perticas tres». Au passage, on remarque dans cet acte que les capita sont beaucoup plus courts que les latera. La nécessité de préciser la longueur des côtés vient peut-être du fait qu'ils sont irréguliers (pour la deuxième parcelle).

${ }^{100}$ AAL 15 (++O64), Bert. 37 (a. 729) : «tabola una recipiente modiolas duodecim».

${ }^{101}$ AAL 37 (++I64), ChLA 929, Bars. 39, CDL 91 (a. 747) : «una iscala de uno laton.

${ }^{102}$ Le muid est la mesure de superficie la plus employée, suivie il est vrai par le setier (sistaria). 
Évolution des systèmes métrologiques

Reste à résoudre le délicat problème du passage des mesures antiques aux mesures en cours aux VIII ${ }^{e}$ et IX $^{e}$ siècles à Lucques. Certaines mesures ont apparemment été conservées telles qu'à leur origine, comme la tabu$l a^{103}$. D'autres portent des appellations similaires, comme la pertica. Dans le cas de Lucques, il existe un lien évident entre la perche et le muid.

Des érudits du $\mathrm{XIX}^{\mathrm{e}}$ siècle se sont longuement penchés sur le problème du glissement d'un système métrologique à l'autre. Il est d'ailleurs rendu plus complexe par le fait que, si le système métrologique change, la structure globale du parcellaire et des champs n'évolue que très peu. On peut tirer de la lecture de ces différentes études les conclusions suivantes :

- On observe d'une part un passage de la pertica romaine (de 10 pieds, soit pertica decimpeda) à la pertica lombarde (de 12 pieds, soit pertica dodecimpeda), et du pied romain au pied lombard, dit de Liutprand (qui correspond en fait au cubitus romain), selon un coefficient de 1,5.

- D'autre part, on se met à calculer le jugère selon un mode différent : le jugère romain est fait d'un rectangle de $12 \times 24$ perches linéaires romaines (soit $120 \times 240$ pieds, donc 28800 pieds carrés, ce qui correspond aussi à 288 perches linéaires carrées) ${ }^{104}$. Le jugère lombard, quant à lui, est calculé selon un schéma géométrique différent : c'est un rectangle composé de 12 perches iugialis ou "pertica da tavola», chaque perche iugialis étant, non pas une perche linéaire carrée, mais un rectangle de côté $1 \times 24$ perches linéaires, soit 24 perches linéaires carrées, appelées aussi tavola ${ }^{105}$. Le jugère fait donc $12 \times 24$ perches linéaires carrées, soit 288 perches linéaires carrées. On arrive, somme toute, au même résultat (en nombre de perches linéaires carrées), mais on se rend compte que les Lombards, au lieu de multiplier simplement, ressentent la nécessité de créer une unité de surface intermédiaire, qui trahit leur incapacité à passer de la mesure linéaire à la mesure de superficie. C'est en tout cas ce que cette construction nous suggère. Au-delà de ce système de construction de la surface, la valeur du jugère, du moins dans le Regnum, est nettement différente, dans la mesure où la valeur de la perche linéaire a complètement changé (d'une

${ }^{103}$ Mesure de surface, représentant dans le système romain un quart de jugère, soit 72 perches carrées.

${ }^{104} \mathrm{Cf}$. De Iugeribus metiundis, op. cit. supra note 84, calculé sous la forme de 18 $\times 16$, ce qui revient au même.

${ }^{105}$ Pour cette composition du jugère, voir V. Villani, art. cit. supra note 92. 
perche de 10 à 12 pieds, et d'un pied romain à un pied lombard). Cela induit une variation énorme de la surface réelle entre un jugère romain et un jugère lombard, ce qui retentit sur le calcul du muid.

Le vocable le plus souvent cité pour désigner le pied lombard, ou pied de Liutprand, est "pes ligitimus", qui gagne ensuite, comme par contamination, la perche : "pertica legiptima, iusta pertica". Or, cette expression apparaît assez rapidement dans le corpus d'actes lucquois ${ }^{106}$. On peut donc penser que les évolutions des systèmes métrologiques, qui prennent naissance au cœur du Regnum lombard, gagnent assez rapidement la zone lucquoise, par ailleurs très liée aux Lombards de la plaine padane ${ }^{107}$.

Calcul des surfaces : le problème du muid

- Enfin, il faut tenter de savoir comment on calcule le muid, qui est la mesure de superficie donnée dans les actes lucquois. On trouve le mode de calculer la valeur du muid à l'époque romaine, notamment dans le De iugeribus metiundis, qui indique aussi son équivalence dans les autres mesures romaines $^{108}$. Dans le système romain, c'est une subdivision du jugère, qui représente un tiers de sa valeur, soit 96 perches carrées, donc 9600 pieds carrés.

Les mesures de superficie lucquoises

Le dernier élément de notre enquête s'appuie sur des indices relevés dans les actes de Lucques. Selon un texte de $847^{109}$, le muid est divisé en 6 coltri, la coltre faisant chez les arpenteurs modernes lucquois 460 perches carrées agrimensorielles. Or, cette mesure est fondée sur une valeur de la perche agrimensorielle lucquoise de 5 braccia, le braccio représentant le double du pied romain : donc la perche agrimensorielle lucquoise correspond très exactement à la perche de 10 pieds romaine. Cette valeur de la coltra permet, selon Mazzi, aux Lucquois, de trouver un bel accomodement entre les mesures romaines et les mesures lombardes ${ }^{110}$. On s'entend

${ }^{106}$ Cf. Cordero di San Quintino, art. cit. supra note 92.

${ }^{107} \mathrm{~V}$. Villani a bien mis en valeur cette extension progressive du système métrologique lombard dans l'ensemble de l'Italie, essentiellement dans les zones d'influence ou de contact politique avec les Lombards.

${ }^{108}$ Voir l'édition du Traité "De iugeribus metiundis", op. cit. supra note 84.

${ }^{109}$ Cité par Cordero di San Quintino, art. cit. supra note 92.

${ }^{110}$ En raison de la complexité de la démonstration de A. Mazzi, Il piede Liprando, qui effectue ce développement dans une longue note p. 115 , je me suis résolue à ne donner que les éléments essentiels de son affirmation. Tout lecteur intéressé pourra s'y reporter pour de plus amples informations. La démonstration de Mazzi semble assez pertinente. Un exposé sur les mesures lucquoises a aussi été donné par 
communément à penser qu'auparavant, le système de mesures employé à Lucques est directement hérité du système romain ${ }^{11}$.

Tout semble donc indiquer que, derrière l'apparente hétérogénéité du système métrologique en vigueur à Lucques aux VIII ${ }^{e}$ et $\mathrm{IX}^{\mathrm{e}}$ siècles, déroutante pour nous, car deux systèmes semblent s'affronter ou se contredire, il existe un système subtil d'équivalences, qui permet aux notaires de jongler entre les deux systèmes métrologiques de référence. Il permet aux notaires de s'exprimer selon la norme métrologique lombarde, qui est celle, aussi, des contractants, mais en gardant la possibilité d'intégrer ces parcelles dans leur cadre d'origine (le réseau romain). Cela permet d'expliquer, peutêtre, la permanence des structures parcellaires, et du découpage en centuries, dans un cadre différent (du moins dans ses représentations). Outre la persistance d'un réseau orthonormé, la filiation entre le système métrologique romain et celui qui est en cours à Lucques aux VIII-IX ${ }^{\mathrm{e}}$ siècles semble permettre aux notaires de décrire les parcelles, y compris d'un point de vue métrologique, en se coulant dans la structure précédente.

Ce système semble en tout état de cause indiquer une certaine habileté des notaires lucquois à manipuler les chiffres et les systèmes métrologiques, preuve, s'il en est besoin, de leur bonne maîtrise des techniques d'arpentage. D'ailleurs, le soin qu'ils apportent à préciser le type de mesure qu'ils utilisent dans les transactions et les opérations de mesure montre bien à quel point ils sont conscients de la nécessité de précision utile à ce type d'opérations.

\section{Formation CULTURELLE DES NOTAIRES}

L'indication d'estimation de surfaces sans précision du mode de calcul pour y arriver pose un problème notable : les notaires lucquois calculaientils réellement la surface des parcelles, ou se livraient-ils à une évaluation approximative? Autrement dit, les notaires savaient-ils multiplier? Il est donc nécessaire d'aborder ici la formation technique et culturelle de ces personnages. Naturellement, leur évolution dans un monde où persistent encore certaines structures romaines nous pousse à savoir quels rapports ils entretenaient avec la culture technique classique, et notamment s'ils connaissaient les textes techniques relatifs à l'arpentage. En fait, c'est ici

G. Cordero di San Quintino, qui donne des conclusions légèrement différentes, mais qui ouvrent aussi sur cette adaptation des mesures lucquoises aux deux systèmes métrologiques de référence.

"II Cf. G. Cordero di San Quintino, art. cit. supra note 92. 
tout le vaste débat de la transmission du savoir technique antique qui est posé. Plusieurs éléments doivent être rappelés pour effectuer un raisonnement serein.

Transmission des savoirs techniques. Circulation des textes gromatiques

Il faut en premier lieu tenter de comprendre quelles étaient les conditions de transmission de ce savoir. En fait, le débat est sous-tendu par une série d'éléments : accepter l'idée qu'il y ait eu une transmission construite, et consciente, de ce savoir durant la période des VI-VIII ${ }^{e}$ siècles revient à réviser nombre de schémas sur les formes de la culture de l'Antiquité tardive et du tout premier Moyen Âge.

La nature des textes gromatiques constitue déjà un élément particulier de la réflexion : il s'agit d'un corpus de textes extrêmement variés, de peu d'ampleur, composés à des époques diverses, et insérés dans des recueils spécialisés, à l'usage d'un personnel administratif disséminé dans l'ensemble de l'Empire. Les textes sont élaborés à leur usage, et comprennent des éléments techniques divers : mesure de l'espace, techniques de marquage, résolution des conflits de bornage... Ils abordent donc des domaines variés, d'ordre fiscal, mais aussi technique (calcul des surfaces, selon des techniques géométriques simples) et juridique. On conçoit aisément une communication de ces diverses connaissances techniques, entre les divers centres urbains dotés de magistrats titulaires de la juridiction.

La multiplication des copies, dès l'époque antique, et leur circulation dans l'ensemble de l'Empire doivent dans ce contexte être une condition favorable à la bonne diffusion de ces textes et à leur connaissance par un nombre relativement important de personnes. Autre élément, et non des moindres : la plupart des recueils de textes gromatiques remonte à une période contenue entre les $\mathrm{V}^{\mathrm{e}}$ et $\mathrm{VII}^{\mathrm{e}}$ siècles (sans exclure le VIII ${ }^{\mathrm{e}}$ siècle). Selon L. Toneatto, le haut Moyen Âge reprend les textes antiques, avec beaucoup d'attention, et produit de nouvelles œuvres, qui semblent des tentatives de réorganiser la matière selon des exigences de diverses natures, parmi lesquelles une reprise des techniques antiques à des fins pratiques, liées à une fonction d'arpentage qui devait encore persister. On assiste ainsi à l'éclosion de recueils réorganisés selon ces exigences. C'est ainsi le cas de l'ars de Gisemundus ${ }^{112}$. On remarque le même type de procédé dans le

112 Voir L. Toneatto, Note sulla tradizione del Corpus agrimensorum Romanorum. I. Contenuti e struttura dell'Ars gromatica di Gisemundus (IXe s.), dans MEFRM, 94, 1982, p. 191-313 et Id., Codices artis mensoriae - I manoscritti degli antichi opuscoli latini d'agrimensura (V-XIX sec.), Spoleto, 1994-1995. 
monde byzantin, qui continue de produire des «manuels» à l'usage des agents fiscaux ${ }^{113}$. La copie des textes semble en grande partie motivée par des considérations d'ordre technique : il s'agit de constituer un corpus de textes pratiques afin de résoudre certains problèmes. On ne se place pas encore dans un contexte de copie à titre de curiosité d'ordre scientifique, ou littéraire : il ne s'agit pas de conserver des textes anciens parce qu'ils sont anciens, mais de les utiliser. Pour le monde byzantin, J. Lefort rappelle qu'il est illusoire d'analyser le contenu de ces manuels pour mesurer la qualité des connaissances mathématiques de l'époque : il s'agit de textes techniques appliqués. Les personnes qui commandent des copies de ces textes attendent un accès à des recettes, ou des réponses à des questions d'ordre juridico-technique (que faire en cas de conflit de limite?).

Les conditions de transmission et de copie du corpus des agrimensores latins, telles que les rétablit $\mathrm{L}$. Toneatto, laissent donc penser que, au début du haut Moyen Âge, ces textes circulaient assez bien dans l'ensemble de l'ancien Empire romain, de sa partie la plus orientale à ses confins occidentaux et septentrionaux. L'ars Gisemundi a ainsi été probablement écrite en Catalogne au début du IX $\mathrm{X}^{\mathrm{e}}$ siècle, et les manuscrits les plus anciens conservés des agrimensores latins ont été copiés dans la partie septentrionale de l'Empire carolingien. On peut penser que les textes techniques relatifs à l'arpentage étaient assez bien connus. Malgré tout, il n'empêche que leur contenu, déjà à cette époque, n'était pas toujours totalement compris et intégré : à preuve, les erreurs de copie du De iugeribus metiundis, signalées par J.-M. Martin et J.-P. Grélois, qui trahissent en fait une méconnaissance de certaines techniques de calcul (et notamment de la multiplication).

Dans l'étude de J. Lefort, on se rend compte que les notaires de l'espace byzantin qui ont commandé des copies de traités de géométrie pratique, qui utilisaient ces manuels d'arpentage et tentaient de calculer des surfaces de parcelles, ne savaient pas toujours multiplier et calculer la surface de trapèzes non isocèles : les algorithmes à suivre, dans ces cas-là, sont copiés avec des erreurs, ce qui fait supposer que les copistes ignoraient tout du mode de calcul proposé.

À partir de ces quelques données, nous devons établir un constat double : d'une part, on peut penser avec beaucoup de probabilité que les textes techniques étaient transmis, et circulaient bien au cours du haut Moyen Âge, et donc qu'il n'y a pas eu de rupture, ou de coupure culturelle brutale entre la période antique et le haut Moyen Âge : ainsi, Isidore de Sé-

${ }^{113}$ Cf. J. Lefort, op. cit. supra note 84 , introduction générale. 
ville, dans bien des passages de son œuvre, utilise des passages des agrimensores latins. D'autre part, cette transmission ne se fait pas sans distorsions et incompréhensions, qui pointent à certains moments. Cela peut expliquer l'emploi d'un vocabulaire et de techniques d'origine antique parfois vidés, ou détournés, de leur sens originel.

Dans le prolongement de ce raisonnement, nous pouvons citer la complexité de construction du jugère lombard, décomposé en petits rectangles ajoutés les uns aux autres. En effet, si les modules correspondent en fait au système métrologique romain (dans leur structure), le mode employé pour arriver au résultat est parfaitement révélateur du processus que nous venons de souligner : on applique une recette, un algorithme nettement lié aux techniques de calcul romaines, mais sans vraiment le comprendre.

De plus, à Lucques, dans les descriptions de biens où sont données de manière précise les mesures de chaque côté, on se rend compte que ceux-ci sont inégaux, ce qui peut laisser penser que l'on fait le contour de la parcelle (en indiquant la longueur des côtés), mais qu'on ne connaît pas sa surface ${ }^{114}$. Mais cet acte, déjà cité, est atypique, et de plus, il a été écrit dans une région marginale, ou hors de la sphère notariale lucquoise. Malgré tout, si l'acte est conservé à l'Archivio, les notaires lucquois avaient plus ou moins connaissance de cette technique de limitation, mais ils employaient de manière privilégiée un autre système.

\section{Formation culturelle des notaires lucquois : le Codex 490}

Quoi qu'il en soit, il convient aussi d'établir si les notaires lucquois avaient accès, peu ou prou, à cet ensemble de textes. Nous avons la grande chance de disposer d'un indicateur extrêmement précieux de la culture des notaires lucquois à la fin du VIII ${ }^{e}$ siècle et au début du IX' siècle. Il s'agit du Codex 490 de l'Archivio Arcivescovile di Lucca, conservé dans les archives mêmes de la cathédrale de Lucques.

Il importe tout d'abord de se pencher sur son contenu : ce codex semble révéler un «bain culturel antique». En effet, les divers éléments qui composent le codex semblent former un recueil de caractère technique, ou thématique, qui devait servir pour ses utilisateurs à calculer le temps : mesure du temps, calendrier agricole, prévision des tempêtes,... Les extraits choisis sont extrêmement divers, puisés dans un "stock» d'auteurs assez varié, de Pline l'Ancien à Isidore de Séville, en passant par des éléments du Liber pontificalis, ou du De natura rerum de Bède pour ne citer que quel-

${ }^{114}$ Cf. supra note 99 , AAL 386. 
ques passages. Les extraits ont été choisis avec soin, pour répondre à la cohérence thématique demandée par le commanditaire, mais disposés selon un ordre qu'il est difficile de déterminer. On peut penser que ce type de copie est à placer dans une tradition technique où la compréhension intrinsèque du texte et de ses caractéristiques techniques, de savoir, importait plus que des considérations d'ordre littéraire (il ne s'agit pas ici de faire œuvre de conservation "gratuite», ou littéraire, de textes antiques). La convergence de textes techniques vers Lucques, à cette occasion, semble indiquer que la cité était intégrée à un réseau de circulation culturel qui comprenait des œuvres récentes (le Liber pontificalis), mais aussi bien plus anciennes (Pline).

Par ailleurs, les conditions d'élaboration de ce codex ont été éclaircies par L. Schiaparelli et A. Petrucci ${ }^{115}$. Ces deux grands spécialistes de l'écriture et de la diplomatique du haut Moyen Âge ont établi avec fermeté deux éléments fondamentaux : la datation et l'identité d'une part de la personne qui a commandé le codex, d'autre part de certains des copistes. Nous pouvons donc établir la fourchette chronologique de copie de cet ensemble de textes : l'ouvrage a été conçu, et les textes copiés, entre 787-796 et 816 , à l'initiative, probablement, d'un des évêques de Lucques, Jean, et de son successeur, Jacques. A. Petrucci conclut à une copie progressive des textes, en fonction de leur réception à l'atelier de copie, et de l'arrivée de scribes supplémentaires. Certains des copistes de ce codex sont des notaires lucquois : leur main a été formellement reconnue par ces deux auteurs. On pense même que l'un des deux évêques a participé matériellement à l'élaboration du codex.

On peut conclure, avec beaucoup de prudence, que les copistes et notaires lucquois avaient donc accès, par quelque chemin que ce soit, à un corpus de textes techniques hérités de l'Antiquité. A. Petrucci semble dessiner des provenances des textes assez diverses, notamment de Rome, mais aussi de l'Espagne. Cela semblerait indiquer que Lucques est bien intégrée aux réseaux de circulation des textes, techniques et littéraires. Pourquoi ne pas penser que les notaires de Lucques (dont on a vu qu'ils exerçaient aussi une fonction de copistes, et qu'ils avaient un niveau culturel suffisant pour assumer une entreprise de copie aussi ambitieuse que celle du Codex

${ }^{115}$ Cf. L. Schiaparelli, Il Codice 490 della Biblioteca capitolare di Lucca (sec. VIIIIX). Contributo allo studio della minuscola precarolina in Italia (Studi e testi, 36), Rome, 1924. A. Petrucci, Il Codice 490 della Biblioteca capitolare di Lucca : un problema di storia della cultura medievale ancora da risolvere, dans Actum Luce, II 2 , Lucques, 1973, p. 159-175. 
$490^{116}$ ) ont eu accès à des copies de textes tirés des agrimensores latins? Cette hypothèse de travail, si elle ne peut être prouvée, peut toutefois être avancée avec quelques motifs sérieux. Il semble que :

- le nombre de copies du corpus d'arpentage romain en circulation était assez important à cette époque, qu'il y en avait en Italie, en Espagne et dans la partie septentrionale de l'Empire carolingien;

- d'autre part, Lucques est placée à la croisée d'importantes voies de circulation : nombre d'éléments convergent donc vers cette hypothèse de travail.

Au terme de ce long raisonnement, il convient de rassembler tous les éléments de notre argumentation. On note à Lucques des structures du parcellaire très profondément conditionnées par les centuriations romaines, en parallèle avec des sytèmes de description du parcellaire qui semblent indiquer une filiation directe avec les traditions antiques. On observe en outre une métrologie qui présente des similitudes avec le système romain. Enfin, le milieu notarial se livre à des opérations de mesures des longueurs, avait potentiellement accès aux textes techniques d'arpentage romain, et donnait des indications de superficies.

Il ne faut pas oublier à ce propos que les parcelles décrites par capita et latera étaient probablement régulières, et de plus situées dans un cadre strictement orthonormé : cela facilite le passage de la mesure linéaire à la mesure de surface ${ }^{177}$. Dans son article, J.-M. Martin écrit qu' «à Naples, on sait passer de la longueur à la superficie, opération qui ne se fait nulle part ailleurs (...) Les curiales traduisent clairement ce passage au moyen d'expressions du genre ad mensura per passos modios quinque». Il rappelle aussi que l'on se situe dans un espace de petites parcelles cultivées, régulières et de taille restreinte, et que d'autre part, la persistance à Naples de certaines traditions romaines ne semble pas étrangère à cette capacité technique. L'examen des chartes fait clairement apparaître que l'espace de la plaine lucquoise est orthonormé, ou pour le moins qu'une grille régulière, héritée des centuriations romaines, est encore en vigueur et structure net-

$116 \mathrm{Je}$ tiens à remercier M.-É. Boutroue, qui m'a fourni avec générosité bon nombre de renseignements sur les conditions de transmission des textes antiques au cours du Moyen Âge et a accepté d'examiner attentivement les passages de Pline contenus dans le Codex 490. Il semble que les copistes aient fait en l'occurrence du "bon travail», et que le gauchissement du texte latin n'ait été que limité, preuve du «bon niveau» culturel des copistes lucquois, si tant est que nous puissions apprécier leur travail selon ce mode.

${ }^{117}$ On peut se reporter ici à l'affirmation de J. Lefort en la matière, op. cit. supra note 84 . 
tement l'espace. Ici, la description de parcelles quadrangulaires ou rectangulaires (allant du carré à la lanière) est suffisamment fréquente pour appuyer cette affirmation. Enfin, nous pouvons avancer que la régularité du parcellaire, encore très nettement visible actuellement, est un indice supplémentaire de cette pérennité ${ }^{118}$. La coïncidence est donc trop forte pour ne pas penser, mutatis mutandis, que le même processus peut être observé à Lucques.

Ainsi, sous cet éclairage technique et culturel, la pratique notariale lucquoise et les systèmes de description du parcellaire témoignent de l'ancrage encore très fort dans des structures héritées du monde antique. Nous ne pouvons affirmer certains points (les notaires, par exemple, ne devaient pas savoir faire des multiplications, opération pourtant nécessaire), mais il semble malgré tout que la partie technique des contrats (évaluation des surfaces, limitations des parcelles, localisation, ...), assumée par les notaires, soit effectuée avec un maximum de précision, en s'appuyant sur un vocabulaire et des techniques d'origine romaine. Si les notaires ne calculent pas formellement les surfaces des parcelles, ils effectuent malgré tout un bon travail d'experts, et ils peuvent peut-être aussi s'appuyer pour ce faire sur le souvenir plus ou moins net des surfaces des parcelles antiques, ou sur un "cadastre", plus ou moins formalisé, encore en cours à cette époque, et hérité lui-même de la période romaine ${ }^{119}$. On peut en outre penser qu'ils se livrent à ces activités dans un but juridique : la limitation permet d'éviter au maximum les conflits de bornage, par exemple. Au-delà de cet aspect, qui semble assez évident, il semble difficile d'affirmer que les opérations de mesure entrent dans un processus d'appréciation de la terre : l'évaluation de la surface n'intervient pas uniquement. Il faut ajouter d'autres critères, d'ordre matériel (qualité de la terre, culture ouvrant à un revenu plus ou moins important), ou socio-économique (enjeu patrimonial, dynamique du marché foncier, facteurs sociaux), qui ne sont pas forcément cités dans les contrats que nous avons examinés.

Le rôle des notaires semble donc fondamental dans ces affaires : dépositaires d'une mémoire foncière très précise, ils jouissent aussi d'un savoir technique et d'une autorité juridique (ce sont eux qui sont chargés d'effectuer des expertises, mais aussi qui valident littéralement les transactions en les enregistrant soigneusement, en les conservant) qui en font les personnages indispensables à la bonne marche de la dynamique foncière de la zone. Au-delà de leur rôle de conservateurs de la mémoire foncière, ils

${ }^{118}$ C'est d'ailleurs grâce à cette pérennité que F. Castagnoli a repéré de manière assez précoce la centuriation de Lucques, et qu'il l'a datée.

${ }^{119}$ J.-M. Martin suggère lui-aussi cette hypothèse. 
jouent un rôle fondamental dans la maîtrise de l'espace : le décrire, c'est aussi en quelque sorte le fixer, le stabiliser. La pose de bornes de confins, ainsi que la protection du bornage, concourent à ce processus. L'indication des surfaces des parcelles dans les actes permet de même d'en fixer les limites et l'extension, leur modification étant de facto contrôlée par l'intermédiaire de procédures juridiques précises, et le plus souvent empêchée ou réprimée. En ce sens, l'activité des notaires peut être comparée à une opération de cadastration (sans visée fiscale, bien évidemment). Si les arpenteurs romains n'existent plus, il semble donc bel et bien que les notaires assument cette fonction.

Anne Mailloux 